\title{
Universiteit
}

Leiden

The Netherlands

\section{Spitzer SAGE-SMC Infrared Photometry of Massive Stars in the Small Magellanic Cloud}

Bonanos, A.Z.; Lennon, D.J.; Köhlinger, F.; Loon, J.T. van; Massa, D.L.; Sewilo, M.; ... ; Whitney, B.A.

\section{Citation}

Bonanos, A. Z., Lennon, D. J., Köhlinger, F., Loon, J. T. van, Massa, D. L., Sewilo, M., ... Whitney, B. A. (2010). Spitzer SAGE-SMC Infrared Photometry of Massive Stars in the Small Magellanic Cloud. The Astronomical Journal, 140(2), 416-429. doi:10.1088/0004-6256/140/2/416

Version: $\quad$ Not Applicable (or Unknown)

License: $\quad$ Leiden University Non-exclusive license

Downloaded from: https://hdl.handle.net/1887/61635

Note: To cite this publication please use the final published version (if applicable). 


\title{
SPITZER SAGE-SMC INFRARED PHOTOMETRY OF MASSIVE STARS IN THE SMALL MAGELLANIC CLOUD
}

\author{
A. Z. Bonanos ${ }^{1}$, D. J. Lennon ${ }^{2,3}$, F. Köhlinger ${ }^{4}$, J. Th. van Loon ${ }^{5}$, D. L. Massa ${ }^{2}$, M. Sewilo ${ }^{2}$, C. J. Evans ${ }^{6}$, N. Panagia ${ }^{2,7,8}$, \\ B. L. Babler ${ }^{9}$, M. Block ${ }^{10}$, S. Bracker $^{9}$, C. W. Engelbracht ${ }^{9}$, K. D. Gordon ${ }^{2}$, J. L. Hora ${ }^{11}$, R. Indebetouw ${ }^{12}$, \\ M. R. Meade ${ }^{9}$, M. MeiXner ${ }^{2}$, K. A. Misselt ${ }^{10}$, T. P. Robitaille ${ }^{11}$, B. Shiao ${ }^{2}$, And B. A. Whitney ${ }^{13}$ \\ ${ }^{1}$ Institute of Astronomy \& Astrophysics, National Observatory of Athens, I. Metaxa \& Vas. Pavlou St., P. Penteli, 15236 Athens, Greece; bonanos@ astro.noa.gr \\ ${ }^{2}$ Space Telescope Science Institute, 3700 San Martin Drive, Baltimore, MD 21218, USA; lennon@stsci.edu, massa@stsci.edu, sewilo@stsci.edu, \\ panagia@stsci.edu,kgordon@stsci.edu, meixner@stsci.edu, shiao@stsci.edu \\ ${ }^{3}$ European Space Agency, Research and Scientific Support Department, Baltimore, MD 21218, USA \\ ${ }^{4}$ Department of Physics and Astronomy, Heidelberg University, Albert-Ueberle-Str. 3-5, D-69120 Heidelberg, Germany; koehlinger@ stud.uni-heidelberg.de \\ ${ }^{5}$ Astrophysics Group, Lennard-Jones Laboratories, Keele University, Staffordshire ST5 5BG, UK; jacco@ astro.keele.ac.uk \\ ${ }^{6}$ UK Astronomy Technology Centre, Royal Observatory Edinburgh, Blackford Hill, Edinburgh, EH9 3HJ, UK; chris.evans@ stfc.ac.uk \\ ${ }^{7}$ INAF/Osservatorio Astrofisico di Catania, Via Santa Sofia 78, I-95123 Catania, Italy \\ ${ }^{8}$ Supernova Ltd., OYV 131, Northsound Road, Virgin Gorda, British Virgin Islands \\ ${ }^{9}$ Department of Astronomy, 475 North Charter Street, University of Wisconsin, Madison, WI 53706, USA; \\ brian@sal.wisc.edu, s_bracker@hotmail.com, meade@astro.wisc.edu \\ ${ }^{10}$ Steward Observatory, University of Arizona, 933 North Cherry Avenue, Tucson, AZ 85721, USA; mblock @ as.arizona.edu, \\ cengelbracht@as.arizona.edu,kmisselt@as.arizona.edu \\ ${ }^{11}$ Harvard-Smithsonian Center for Astrophysics, 60 Garden Street, MS 67, Cambridge, MA 02138, USA; jhora@cfa.harvard.edu, trobitaille@cfa.harvard.edu \\ ${ }^{12}$ Department of Astronomy, University of Virginia, P.O. Box 3818, Charlottesville, VA 22903, USA; remy@ virginia.edu \\ ${ }^{13}$ Space Science Institute, 4750 Walnut Street, Suite 205, Boulder, CO 80301, USA; bwhitney@ spacescience.org \\ Received 2010 April 6; accepted 2010 June 2; published 2010 June 24
}

\begin{abstract}
We present a catalog of 5324 massive stars in the Small Magellanic Cloud (SMC), with accurate spectral types compiled from the literature, and a photometric catalog for a subset of 3654 of these stars, with the goal of exploring their infrared properties. The photometric catalog consists of stars with infrared counterparts in the Spitzer SAGE-SMC survey database, for which we present uniform photometry from 0.3 to $24 \mu \mathrm{m}$ in the $U B V I J H K_{s}+$ IRAC+MIPS24 bands. We compare the color-magnitude diagrams and color-color diagrams to those of stars in the Large Magellanic Cloud (LMC), finding that the brightest infrared sources in the SMC are also the red supergiants, supergiant $\mathrm{B}[\mathrm{e}](\mathrm{sgB}[\mathrm{e}])$ stars, luminous blue variables, and Wolf-Rayet stars, with the latter exhibiting less infrared excess, the red supergiants being less dusty and the $\mathrm{sgB}[\mathrm{e}]$ stars being on average less luminous. Among the objects detected at $24 \mu \mathrm{m}$ in the SMC are a few very luminous hypergiants, four B-type stars with peculiar, flat spectral energy distributions, and all three known luminous blue variables. We detect a distinct $\mathrm{Be}$ star sequence, displaced to the red, and suggest a novel method of confirming Be star candidates photometrically. We find a higher fraction of Oe and Be stars among $\mathrm{O}$ and early-B stars in our SMC catalog, respectively, when compared to the LMC catalog, and that the SMC Be stars occur at higher luminosities. We estimate mass-loss rates for the red supergiants, confirming the correlation with luminosity even at the metallicity of the SMC. Finally, we confirm the new class of stars displaying composite A \& F type spectra, the $\mathrm{sgB}$ [e] nature of 2dFS1804 and find the F0 supergiant 2dFS3528 to be a candidate luminous blue variable with cold dust.
\end{abstract}

Key words: catalogs - galaxies: individual (SMC) - infrared: stars - stars: early-type - stars: emission-line, Be stars: massive

Online-only material: color figures, machine-readable and VO tables

\section{INTRODUCTION}

The Spitzer Space Telescope Legacy Surveys SAGE (Meixner et al. 2006) and SAGE-SMC (K. D. Gordon et al. 2010, in preparation) have made a comparative study of the infrared properties of massive stars at a range of metallicities possible for the first time by imaging the Large and Small Magellanic Clouds (LMC and SMC). In Bonanos et al. (2009, hereafter Paper I), we presented infrared properties of massive stars in the LMC (at $0.5 Z_{\odot}$ ). The motivation for that study was twofold: (1) to use the infrared excesses of massive stars to probe their winds, circumstellar gas, and dust, and (2) to provide a template for studies of other, more distant, galaxies. Paper I was the first major compilation of accurate spectral types and multi-band photometry from 0.3 to $24 \mu \mathrm{m}$ for massive stars in any galaxy, increasing by an order of magnitude the number of massive stars for which mid-infrared photometry was available. The recently completed SAGE-SMC survey offers the opportunity to extend the study of infrared properties of massive stars to a metallicity of approximately $0.2 Z_{\odot}$ (see, e.g., Hunter et al. 2007) and enables the investigation of their dependence on metallicity, at least over the range $0.2-0.5 Z_{\odot}$.

Infrared excess in hot massive stars is primarily due to free-free emission from their ionized, line-driven, stellar winds. Panagia \& Felli (1975) and Wright \& Barlow (1975) first computed the free-free emission from ionized envelopes of hot massive stars, as a function of the mass-loss rate $(\dot{M})$ and the terminal velocity of the wind $\left(v_{\infty}\right)$. The properties of massive stars, and in particular their stellar winds (which affect their evolution), are expected to depend on metallicity (Z). For example, Mokiem et al. (2007) found empirically that mass-loss rates scale as $\dot{M} \sim Z^{0.83 \pm 0.16}$, in good agreement 
with theoretical predictions (Vink et al. 2001). The expectation, therefore, is that the infrared excesses of OB stars in the SMC should be lower than in the LMC, given that $\dot{M}$ is lower in the SMC. ${ }^{14}$ Furthermore, there is strong evidence that the fraction of classical Be stars among B-type stars is higher at lower metallicity (possibly due to faster rotation, as measured by Martayan et al. 2007b). Grebel et al. (1992) were the first to find evidence for this, by showing that the cluster NGC 330 in the SMC has the largest fraction of Be stars of any known cluster in the Galaxy, LMC or SMC. More recent spectroscopic surveys (Martayan et al. 2010) have reinforced this result. In Paper I, we showed that the Be stars in the LMC are easily discriminated by their mid-infrared colors; we are therefore interested in a comparison with the SMC to quantify the global dependence of the Be star fraction on metallicity. The incidence of $\mathrm{Be} / \mathrm{X}$-ray binaries is also much higher in the SMC than in the LMC (Liu et al. 2005), while the incidence of Wolf-Rayet (W-R) stars is much lower; therefore, a comparison of infrared excesses for these objects is also of interest. Finally, there is interest in the metallicity dependence of the mid-infrared colors of red supergiants, which probe circumstellar dust.

Following the same strategy as in Paper I, we compiled a catalog of massive stars, which we cross-matched in the SAGESMC database to study their infrared properties. This paper is organized as follows. Section 2 describes our spectroscopic and photometric catalogs of massive stars in the SMC, Section 3 presents the resulting color-magnitude and two-color diagrams, and Section 4 presents the infrared excesses detected in various types of massive stars. Section 5 investigates the mass-loss rates in red supergiants and Section 6 summarizes our results.

\section{CATALOG OF MASSIVE STARS IN THE SMC}

As in Paper I, we have compiled a catalog of massive stars with known spectral types in the SMC from the literature. We then cross-matched the stars in the SAGE-SMC database, after incorporating optical and near-infrared photometry from recent surveys of the SMC. The resulting photometric catalog was used to study the infrared properties of the stars. In Section 2.1, we describe the spectral-type catalog compiled from the literature, in Section 2.2, the existing optical, near-infrared, and mid-infrared surveys of the SMC, which were included in the SAGE-SMC database, and in Section 2.3, the cross-matching procedure and the resulting photometric catalog.

\subsection{Spectral-type Catalog}

The criteria for inclusion in the spectral-type catalog of massive SMC stars were the availability of accurate coordinates and accurate (mainly optical) spectral classifications that correspond to stars with masses $\gtrsim 8 M_{\odot}$. The $2 \mathrm{dF}$ Survey ( $2 \mathrm{dFS}$; Evans et al. 2004a, 4161 objects) comprises the largest catalog of SMC stars with spectroscopic classifications and therefore makes up the backbone of our spectral-type catalog. ${ }^{15}$ Previous compilations (e.g., Azzopardi et al. 1975; Azzopardi \& Vigneau 1979, 1982) were included with updated coordinates from Brian Skiff's lists ${ }^{16}$ and spectral types from the literature, when available. The literature search resulted in 5324 entries. We do not claim completeness; however, we have targeted not only studies

\footnotetext{
${ }_{14}$ The dependence of $v_{\infty}$ on $Z$ is negligible for this range of $Z$ (see Leitherer et al. 1992; Evans et al. 2004b).

15 By retaining all SMC stars from 2dFS, we are possibly including some stars with masses $<8 M_{\odot}$.

$16 \mathrm{ftp}: / / \mathrm{ftp}$. lowell.edu/pub/bas/starcats/
}

Table 1

Catalog of Spectral Types for 5324 SMC Massive Stars

\begin{tabular}{ccccc}
\hline \hline $\begin{array}{c}\text { Star } \\
\text { Name }^{\text {a }}\end{array}$ & $\begin{array}{c}\text { R.A. (J2000) } \\
(\mathrm{deg})\end{array}$ & $\begin{array}{c}\text { Decl. (J2000) } \\
(\mathrm{deg})\end{array}$ & Reference $^{\mathrm{b}}$ & $\begin{array}{c}\text { Classification } \\
\text { \& Comments }\end{array}$ \\
\hline 2dFS0001 & 6.944625 & -73.27911 & E04 & O9.5: \\
2dFS0002 & 7.036375 & -73.49611 & E04 & B0-5V \\
2dFS0003 & 7.089875 & -73.17531 & E04 & B0-5V \\
2dFS0004 & 7.102458 & -73.88650 & E04 & B0-5III \\
2dFS0005 & 7.137542 & -73.30739 & E04 & B0-5V \\
2dFS0006 & 7.243000 & -73.46847 & E04 & A2II \\
2dFS0007 & 7.246125 & -73.28139 & E04 & B1-3II \\
2dFS0008 & 7.322250 & -73.14731 & E04 & B0-5IV \\
\hline
\end{tabular}

Notes.

${ }^{a}$ Star designations: Evans et al. (2dFS; 2004a), Azzopardi et al. (AzV; 1975); Azzopardi \& Vigneau (AzV; 1979, 1982), Feast et al. (R; 1960), Sanduleak (Sk; 1968, 1969), Massey et al. (MPG; 1989).

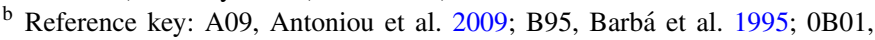
Bartzakos et al. 2001; F03, Foellmi et al. 2003; E04, Evans et al. 2004a; E06, Evans et al. 2006; E10, I. Hunter et al. 2010, in preparation; GC87, Garmany et al. 1987; H83, Humphreys 1983; H03, Harries et al. 2003; H05, Hilditch et al. 2005; L97, Lennon 1997; L06, Levesque et al. 2006; L07, Levesque et al. 2007; MPG89, Massey et al. 1989; M95, Massey et al. 1995; M00, Massey et al. 2000; M02, Massey 2002; MO03, Massey \& Olsen 2003; M03, Massey et al. 2003; M05, Massey et al. 2005; M06, Mennickent et al. 2006; M07, Martayan et al. 2007a, 2007b; M09, Massey et al. 2009; P08, Prieto et al. 2008; R05, Raguzova \& Popov 2005; SB97, Smith Neubig \& Bruhweiler 1997; W07, Wisniewski et al. 2007; Z06, Zickgraf 2006.

(This table is available in its entirety in machine-readable and Virtual Observatory (VO) forms in the online journal. A portion is shown here for guidance regarding its form and content.)

of hot massive stars (defined by their spectral types) in individual clusters and OB associations, but also studies of particular types of massive stars. The largest of these include the survey of $\mathrm{B}$ and Be stars in NGC 330 (Martayan et al. 2007a, 2007b, 314 stars), the VLT-FLAMES Survey of Massive Stars in NGC 330 and NGC 346 (Evans et al. 2006, 232 stars), and unpublished additional FLAMES observations in NGC 346 (PI: Evans; I. Hunter et al. 2010, in preparation, 244 OB \& A-type stars). We have also added the three known luminous blue variables (LBVs), five supergiant B [e] stars (Zickgraf 2006), 69 Be/X-ray binaries (with spectral types from the updated online catalog ${ }^{17}$ of Raguzova \& Popov 2005), early-type eclipsing and spectroscopic binaries, and red supergiants (Massey \& Olsen 2003; Levesque et al. 2006, 2007, 107 stars). The completeness of the catalog depends on the spectral type, e.g., it is $\sim 4 \%$ for the unevolved $\mathrm{O}$ stars in our catalog (out of an estimated total of 2800 unevolved stars with masses $>20 M_{\odot}$ in the SMC; Massey 2010), while the W-R catalog (Massey et al. 2003) is thought to be close to complete.

Table 1 presents our catalog of 5324 massive stars, sorted by R.A., listing: the star name(s), coordinates in degrees (J2000.0), the reference, and corresponding spectral classification. The names of the stars are taken from the corresponding reference. The spectral classifications in the catalog are typically accurate to one spectral type and one luminosity class. We retained about a dozen stars from the above studies with only approximate spectral types (e.g., "early B"). For double entries, we selected the most precise spectral classification available, usually corresponding to the most recent reference.

\footnotetext{
17 http://xray.sai.msu.ru/ raguzova/BeXcat/
} 


\subsection{Optical and Infrared Surveys of the SMC}

Several large optical and infrared photometric catalogs of the SMC have recently become available, enabling us to obtain accurate photometry for its massive star population in the wavelength range $0.3-8 \mu \mathrm{m}$ and in some cases up to $24 \mu \mathrm{m}$. The optical surveys are as follows: the $U B V R$ catalog of Massey (2002) with 85,000 bright stars in an area covering 7.2 $\mathrm{deg}^{2}$, the UBVI Magellanic Clouds Photometric Survey (MCPS; Zaritsky et al. 2002) including 5.1 million stars in the central $18 \mathrm{deg}^{2}$ of the SMC, and the OGLE III catalog containing VI photometry of 6.2 million stars covering $14 \mathrm{deg}^{2}$ (Udalski et al. 2008). The angular resolution is $\sim 2$ ". 6 for the catalog of Massey (2002), $\sim 1^{\prime \prime} .5$ for MCPS, and $\sim 1^{\prime \prime} .2$ for OGLE III.

The existing near-infrared photometric catalogs include: the Two Micron All Sky Survey (2MASS; Skrutskie et al. 2006, extended by 6X2MASS) and the targeted IRSF survey (Kato et al. 2007), which contains 2.8 million sources in the central $11 \mathrm{deg}^{2}$ of the SMC. 2MASS has a pixel scale of $2^{\prime \prime} .0$ pixel $^{-1}$, an average seeing of $2^{\prime \prime} .5$ and limiting magnitudes of $J=15.8$, $H=15.1$, and $K_{s}=14.3$. IRSF has a pixel scale of $0^{\prime \prime} 45$ pixel $^{-1}$, average seeing of $1^{\prime \prime} .3,1^{\prime \prime} .2$, and $1^{\prime \prime} .1$ in the $J H K_{s}$ bands, respectively, and limiting magnitudes of $J=18.8, H=17.8$, and $K_{s}=16.6$. In the mid-infrared, the Spitzer SAGE-SMC survey uniformly imaged the whole SMC $\left(\sim 30 \mathrm{deg}^{2}\right)$ in the IRAC and MIPS bands on two epochs in 2007-2008 (K. D. Gordon et al. 2010, in preparation). The survey has recently produced a combined mosaic catalog of 1.2 million sources. IRAC, with a pixel scale of $1^{\prime \prime} .2$ pixel $^{-1}$, yields an angular resolution of $1^{\prime \prime} .7-2.0$ and MIPS at $24 \mu \mathrm{m}$ has a resolution of $6^{\prime \prime}$.

Given the variation in the depth, resolution, and spatial coverage of these surveys, we included the available photometry for the massive stars in our catalog from all the MCPS, OGLE III, 2MASS, IRSF, and SAGE-SMC catalogs. MCPS has incorporated the catalog of Massey (2002) for bright stars common to both catalogs. Photometry of higher accuracy, particularly in the optical, exists in the literature for many of the stars in our catalog; however, it was not included in favor of uniformity.

\subsection{Photometric Catalog}

\subsubsection{Matching Procedure}

We used the following SAGE-SMC data products to search for the mid-infrared counterparts to the 5324 massive stars listed in Table 1: the v1.0 IRAC Catalog containing IRAC Epoch 1 data bandmerged with All-Sky 2MASS and 6X2MASS catalogs, and the MIPS $24 \mu \mathrm{m}$ Epoch 1 Catalog. The details about the SAGESMC data processing and Legacy data products can be found in K. D. Gordon et al. (2010, in preparation) and in "The SAGESMC Data Description: Delivery 1" document available at the NASA/IPAC Infrared Science Archive Web site. ${ }^{18}$ The IRAC catalog and MIPS $24 \mu \mathrm{m}$ catalog contain high-reliability sources and are subsets of more complete, but less reliable source lists: the IRAC Archive and MIPS $24 \mu \mathrm{m}$ full list (see the data delivery document for a comparison between the catalogs and archives/ full lists).

We started constructing the photometric catalog for the massive stars in our list by selecting their SAGE-SMC IRAC Epoch 1 Catalog counterparts. We performed a conservative neighbor search with a $1^{\prime \prime}$ search radius and selected the closest match

\footnotetext{
18 http://irsa.ipac.caltech.edu/data/SPITZER/SAGE/
}

Table 2

Statistics for the 3654 Matched Stars

\begin{tabular}{lr}
\hline \hline \multicolumn{1}{c}{ Catalogs Matched } & Stars \\
\hline IRACC & 40 \\
IRACC+IRSF & 214 \\
IRACC+IRSF+OGLE & 352 \\
IRACC+MCPS & 3 \\
IRACC+MCPS+IRSF & 57 \\
IRACC+MCPS+IRSF+OGLE & 2733 \\
IRACC+MCPS+OGLE & 54 \\
IRACC+MCPS+MIPS24 & 3 \\
IRACC+MIPS24 & 6 \\
IRACC+MIPS24+IRSF & 35 \\
IRACC+MIPS24+IRSF+OGLE & 10 \\
IRACC+MIPS24+MCPS+IRSF & 80 \\
IRACC+MIPS24+MCPS+IRSF+OGLE & 32 \\
IRACC+OGLE & 35 \\
\hline
\end{tabular}

for each source. We found mid-infrared counterparts for 3654 out of 5324 sources. The IRAC Epoch 1 catalog (IRACC), MIPS $24 \mu \mathrm{m}$ catalog, IRSF, MCPS, and OGLE III catalogs were crossed-matched in the SAGE-SMC database ${ }^{19}$ to provide photometry for sources over a wavelength range from 0.3 to $24 \mu \mathrm{m}$. We used this "universal catalog" to extract multiwavelength photometry for IRAC sources matched to the massive stars. Specifically, for IRAC sources with one or more matches in other catalogs (all but 5), we only considered the closest matches between sources from any two available catalogs (IRAC-MIPS24, IRAC-MCPS, MIPS24-MCPS, IRAC-IRSF, MIPS24-IRSF, IRAC-OGLE III, MIPS24-OGLE III), with distances between the matched sources of $\leqslant 1^{\prime \prime}$. For example, for a match between the IRAC, MIPS $24 \mu \mathrm{m}$, and MCPS catalogs (IRAC-MIPS24-MCPS), we applied these constraints on the IRAC-MIPS24, MIPS24-MCPS, and IRAC-MCPS matches. If the match was not the closest one or the distance was $>1^{\prime \prime}$, we dropped the pair. These stringent criteria were used to ensure that sources from individual catalogs for each multi-catalog match refer to the same star. Table 2 shows the breakdown of the matched stars to the catalogs, such that 40 stars were only matched to the IRACC, 214 only to the IRACC+IRSF catalogs, etc.

\subsubsection{Catalog Description}

Table 3 presents our final matched catalog of 3654 stars, with the star name, IRAC designation, UBVIJHK $K_{s}+$ IRAC+MIPS24 photometry and errors, reference paper, corresponding spectral classification and comments, sorted by increasing R.A. Overall, the photometry is presented in order of shortest to longest wavelength. The 17 columns of photometry are presented in the following order: $U B V I$ from MCPS, $V I$ from OGLE III, $J H K_{s}$ from 2MASS, $J H K_{s}$ from IRSF, IRAC 3.6, 4.5, 5.8, $8.0 \mu \mathrm{m}$ (or [3.6], [4.5], [5.8], [8.0]), and MIPS $24 \mu \mathrm{m}$ (or [24]). A column with the associated error follows each measurement, except for the VI photometry from OGLE III. Henceforth, $J H K_{s}$ magnitudes refer to 2MASS photometry, whereas IRSF photometry is denoted by a subscript, e.g., $J_{\text {IRSF. }}$ All magnitudes are calibrated relative to Vega (e.g., see Reach et al. 2005, for IRAC). In Table 4, we summarize the characteristics of each filter: the effective wavelength $\lambda_{\text {eff }}$, zero magnitude flux (in Jy), angular resolution, and the number of detected stars in each filter.

\footnotetext{
19 http://mastweb.stsci.edu/scasjobs/
} 
Table 3

$0.3-24 \mu \mathrm{m}$ Photometry of 3654 Massive Stars in the SMC

\begin{tabular}{|c|c|c|c|c|c|c|c|c|c|c|}
\hline Star Name $^{a}$ & IRAC Designation & R.A. (J2000) & Decl. (J2000) & $U$ & $\sigma_{U}$ & $B$ & $\sigma_{B}$ & $\ldots$ & Reference $^{b}$ & Classification \& Comments \\
\hline $2 \mathrm{dFS} 0005$ & J002832.82 - 731827.0 & 7.137542 & -73.30739 & 16.33 & 0.042 & 17.063 & 0.023 & $\ldots$ & E04 & $\mathrm{B} 0-5 \mathrm{~V}$ \\
\hline 2dFS0006 & J002858.15 - 732807.0 & 7.243 & -73.46848 & 16.648 & 0.048 & 16.605 & 0.056 & $\ldots$ & E04 & A2II \\
\hline $2 \mathrm{dFS} 0010$ & J002923.69- 734424.6 & 7.349375 & -73.73997 & 16.706 & 0.041 & 17.318 & 0.033 & $\ldots$ & E04 & $\mathrm{B} 0-5 \mathrm{~V}$ \\
\hline 2dFS0019 & J003049.36 - 740000.3 & 7.706292 & -73.99995 & 16.006 & 0.05 & 16 & 0.041 & $\ldots$ & E04 & A0II \\
\hline $2 \mathrm{dFS} 0024$ & J003107.03 - 732709.9 & 7.780042 & -73.45267 & 16.611 & 0.114 & 17.119 & 0.034 & $\ldots$ & E04 & B3IV \\
\hline 2dFS0026 & J003111.52 - 735936.1 & 7.798458 & -73.99333 & 15.744 & 0.049 & 16.292 & 0.033 & $\ldots$ & E04 & $\mathrm{B} 1-3 \mathrm{~V}$ \\
\hline
\end{tabular}

Notes.

a Star designations: Evans et al. (2dFS; 2004a), Azzopardi et al. (AzV; 1975); Azzopardi \& Vigneau (AzV; 1979, 1982), Feast et al. (R; 1960), Sanduleak (Sk; 1968, 1969), and Massey et al. (MPG; 1989).

b Reference key: B95, Barbá et al. 1995; B01, Bartzakos et al. 2001; E04, Evans et al. 2004a; E06, Evans et al. 2006; E10, I. Hunter et al. 2010, in preparation; F03, Foellmi et al. 2003; GC87, Garmany et al. 1987; H83, Humphreys 1983; H03, Harries et al. 2003; H05, Hilditch et al. 2005; L97, Lennon 1997; L06, Levesque et al. 2006; L07, Levesque et al. 2007; MPG89, Massey et al. 1989; M95, Massey et al. 1995; M02, Massey 2002; MO03, Massey \& Olsen 2003; M06, Mennickent et al. 2006; M07, Martayan et al. 2007a, 2007b; M09, Massey et al. 2009; P08, Prieto et al. 2008; R05, Raguzova \& Popov 2005; SB97, Smith Neubig \& Bruhweiler 1997; W07, Wisniewski et al. 2007; Z06, Zickgraf 2006.

(This table is available in its entirety in machine-readable and Virtual Observatory (VO) forms in the online journal. A portion is shown here for guidance regarding its form and content.)

Table 4

Filter and Detection Characteristics

\begin{tabular}{llccc}
\hline \hline Filter & $\begin{array}{c}\lambda_{\text {eff }} \\
(\mu \mathrm{m})\end{array}$ & $\begin{array}{c}\text { Zero Mag } \\
\text { Flux }(\mathrm{Jy})\end{array}$ & $\begin{array}{c}\text { Resolution } \\
\left({ }^{\prime \prime}\right)\end{array}$ & $\begin{array}{c}\text { Stars } \\
\text { Detected }\end{array}$ \\
\hline$U$ & 0.36 & 1790 & $1.5 / 2.6$ & 2921 \\
$B$ & 0.44 & 4063 & $1.5 / 2.6$ & 2962 \\
$V$ & 0.555 & 3636 & $1.5 / 2.6$ & 2962 \\
$I$ & 0.79 & 2416 & $1.5 / 2.6$ & 2844 \\
$V_{\text {OGLE }}$ & 0.555 & 3636 & 1.2 & 3152 \\
$I_{\text {OGLE }}$ & 0.79 & 2416 & 1.2 & 3166 \\
$J$ & 1.235 & 1594 & 2.5 & 3039 \\
$H$ & 1.662 & 1024 & 2.5 & 3007 \\
$K_{S}$ & 2.159 & 666.7 & 2.5 & 2789 \\
$J_{\text {IRSF }}$ & 1.235 & 1594 & 1.3 & 3449 \\
$H_{\text {IRSF }}$ & 1.662 & 1024 & 1.3 & 3413 \\
$K s_{\text {IRSF }}$ & 2.159 & 666.7 & 1.3 & 3345 \\
{$[3.6]$} & 3.55 & 280.9 & 1.7 & 3509 \\
{$[4.5]$} & 4.493 & 179.7 & 1.7 & 3111 \\
{$[5.8]$} & 5.731 & 115.0 & 1.9 & 1038 \\
{$[8.0]$} & 7.872 & 64.13 & 2 & 509 \\
{$[24]$} & 23.68 & 7.14 & 6 & 166 \\
\hline
\end{tabular}

The spatial distribution of our 3654 matched sources is shown in Figure 1, overlaid onto the $8 \mu \mathrm{m}$ image of the SMC. For clarity, only a third of the early- and late-B stars are shown; these are equally distributed along the SMC bar and wing. Other types are concentrated along the bar, partly due to the fact that most spectroscopic surveys have targeted the bar. The $8 \mu \mathrm{m}$ emission, which maps the surface density of the interstellar medium, is stronger in the SMC bar than the wing, since the former contains more star-forming regions.

\section{INFRARED COLOR-MAGNITUDE AND TWO-COLOR DIAGRAMS}

We divide the matched stars into 10 categories according to their spectral types: $\mathrm{O}$ stars, early $(\mathrm{B} 0-\mathrm{B} 2.5)$ and late (B3-B9) B stars (the majority of these have supergiant or giant luminosity classifications), spectral-type A-, F-, and G-type (AFG) supergiants, $\mathrm{K}$ and $\mathrm{M}$ red supergiants (RSGs), W-R stars, supergiant $\mathrm{B}[\mathrm{e}](\mathrm{sgB}[\mathrm{e}]$ ) stars, confirmed LBVs, Be/X-ray binaries and stars with composite features of both A and F stars (AFcomp; Evans et al. 2004a, see also Section 4.4). Note that 813 stars not included in any of the above categories are classified as "other" (and only shown in certain plots). ${ }^{20}$ Most are A stars with luminosity class II or F/G stars without a luminosity classification from Evans et al. (2004a), although with $v_{\text {radial }}>100 \mathrm{~km} \mathrm{~s}^{-1}$. To allow for a direct comparison with the LMC results of Paper I, we present the same color-magnitude and two-color diagrams (CMDs and TCDs), identifying stars in the 10 categories described above. ${ }^{21}$

Infrared [3.6] versus [3.6] - [4.5] and $J-$ [3.6] CMDs for all the stars in the catalog are shown in Figures 2 and 3. The locations of all the SAGE-SMC catalog detections are represented by the gray two-dimensional histogram (Hess diagram). The red giants form the clump at [3.6] > $15 \mathrm{mag}$, while the vertical blue extension contains late-type SMC and foreground stars (free-free emission causes the OB stars to have redder colors). The asymptotic giant branch stars are located at [3.6] $\sim 11$ mag and $J-[3.6] \sim 2$ mag. The RSGs, sgB[e], and LBVs are among the brightest stars at $3.6 \mu \mathrm{m}$ and occupy distinct regions in the diagrams (as in the LMC). Most of the $\mathrm{O}$ and B stars are located along a vertical line at [3.6] $-[4.5] \sim 0$, as expected. The RSGs have "blue" colors because of the depression at [4.5] due to the $\mathrm{CO}$ band (see, e.g., Verhoelst et al. 2009). The $\mathrm{sgB}[\mathrm{e}]$ stars are 0.6-0.8 mag redder and can have similar brightnesses to the RSGs. The late-B stars are brighter than the early-B stars because most of the former are luminous supergiants. A reddening vector for $E(B-V)=0.2 \mathrm{mag}$ (corresponding to some of the largest values for color excess found by Massey et al. 1995; Larsen et al. 2000) is shown in Figure 3 to illustrate the small effect of reddening, which decreases at longer wavelengths. We note that the reddest of the bright AFG supergiants is the F0I eclipsing binary R47 (Prieto et al. 2008). In Figure 3, we find three early-B-type stars with colors similar to the $\mathrm{sgB}[\mathrm{e}] \mathrm{stars}$, although they are fainter at [3.6]. In order of increasing $J-$ [3.6] color, these stars are 2dFS0402, 2dFS2411, and 2dFS2673 (all classified as B0-5V). Their evolutionary status is not clear though they are worthy of

\footnotetext{
${ }^{20}$ Note that five Bextr, five $\mathrm{Be}$, one $\mathrm{Be}$ (Fe II), one Bpec, and one A7-F5e star are found among these.

21 The conversion to absolute magnitudes in all CMDs is based on a true SMC
} distance modulus of $18.91 \mathrm{mag}$ (Hilditch et al. 2005). 


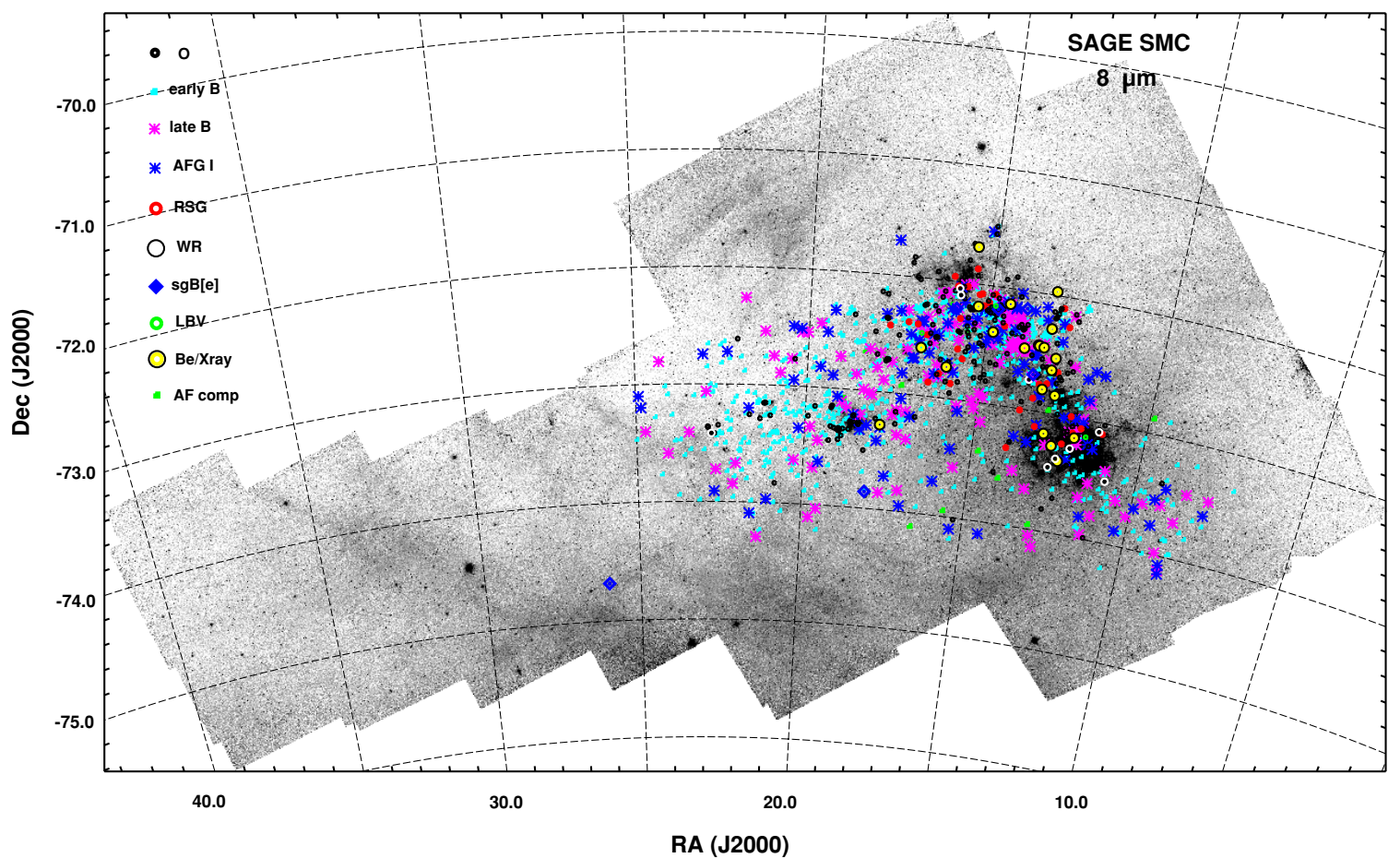

Figure 1. Spatial distribution of massive stars with IRAC counterparts, overplotted onto the $8 \mu \mathrm{m}$ SAGE image (R.A. and decl. are given in degrees). Different symbols denote different spectral types. For clarity, only a third of the early- and late-B stars are shown; these are equally distributed along the SMC bar and wing. Other types are concentrated along the bar, due to the fact that most spectroscopic surveys have targeted the bar.

(A color version of this figure is available in the online journal.)

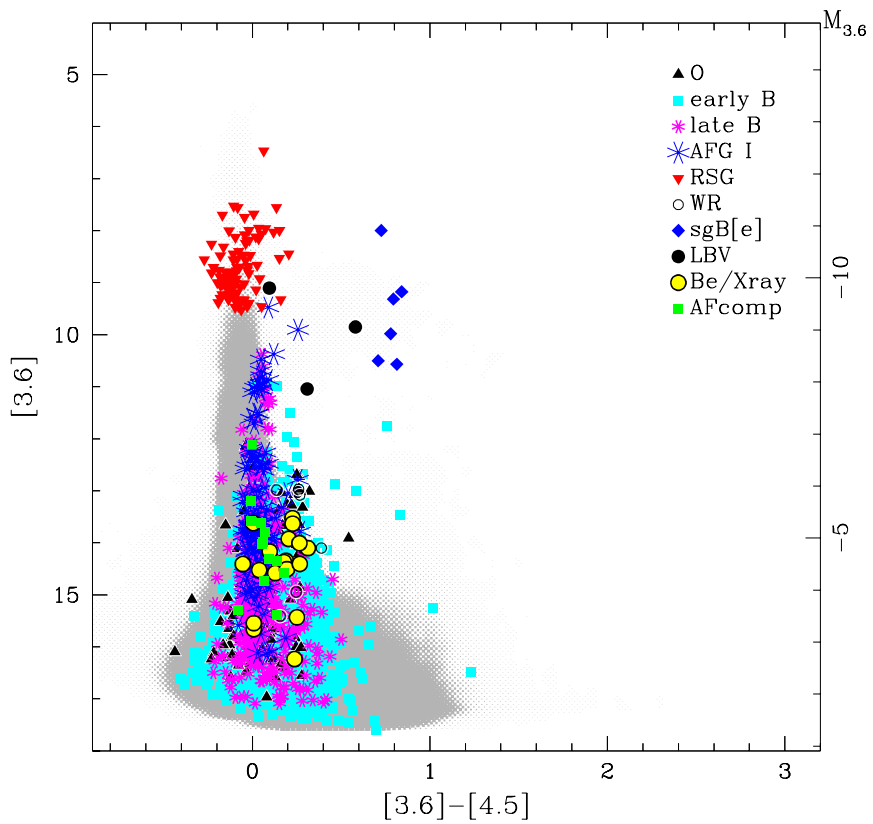

Figure 2. [3.6] vs. [3.6] - [4.5] color-magnitude diagram for massive stars with IRAC counterparts in the SAGE database. The conversion to absolute magnitudes is based on a true SMC distance modulus of 18.91 mag (Hilditch et al. 2005). Different symbols denote different spectral types. The locations of all the SAGE detections are shown in gray as a Hess diagram. The RSGs, $\mathrm{sgB}[\mathrm{e}]$, and LBVs are among the most luminous stars at $3.6 \mu \mathrm{m}$.

(A color version of this figure is available in the online journal.)

future study (see discussion in Section 4.5). A comparison with the LMC CMDs reveals that $\operatorname{sgB}[\mathrm{e}]$ stars are on average less luminous, while the RSGs are less dusty (see Section 5).

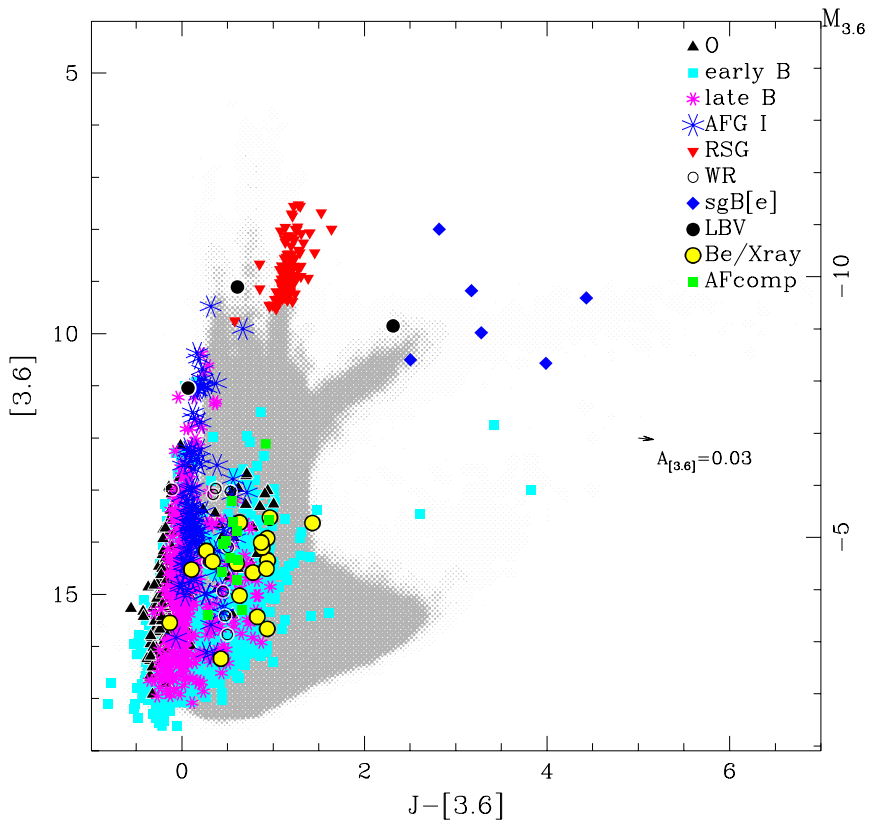

Figure 3. Same as Figure 2, but for the [3.6] vs. $J-[3.6]$ color-magnitude diagram. The reddening vector for $E(B-V)=0.2 \mathrm{mag}$ is shown. The longer baseline separates the populations more clearly.

(A color version of this figure is available in the online journal.)

In Figures 4 and 5, we present [8.0] and [24] versus [8.0] - [24] CMDs, respectively. At these wavelengths, only stars with strong [24] emission are detected, as the sensitivity of Spitzer drops sharply, while the flux from hot stellar photospheres also decreases. Stars without cool dust are located at 


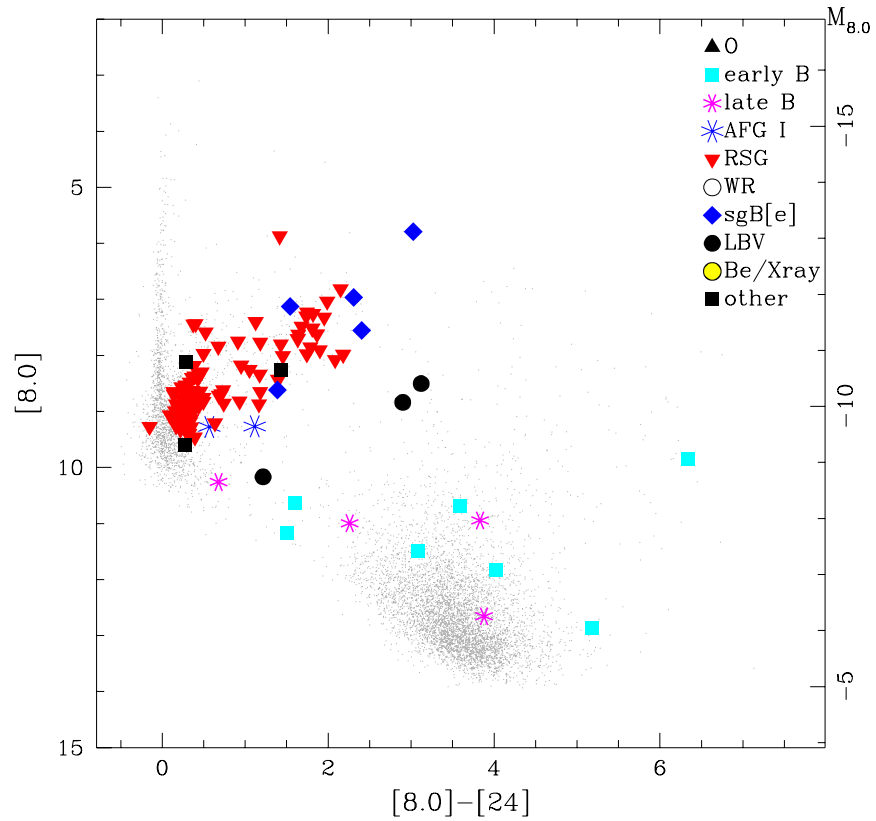

Figure 4. [8.0] vs. [8.0]- [24] color-magnitude diagram, with SAGE detections shown as gray points. The RSGs, sgB[e], and LBVs are also among the most luminous stars at $8 \mu \mathrm{m}$. The names and classifications of stars labeled as "other" are (in order of increasing [8.0] mag): 2dFS0712 (G8), 2dFS3528 (F0), and 2dFS1829 (G2).

(A color version of this figure is available in the online journal.)

[8.0] - [24] 0, while dusty stars (including RSGs, LBVs, $\mathrm{sgB}[\mathrm{e}] \mathrm{stars})$ are reddened. The faint population of sources with [8.0] - [24] $3.5 \mathrm{mag}$ corresponds to background galaxies. We detect dusty LBVs, sgB[e], RSGs, yellow supergiants, and some dusty early-B stars in these CMDs. The detection of the following stars-labeled as "other"-at [24] (in order of increasing magnitude at [24]): $2 \mathrm{dFS} 3528^{22}$ (F0), $2 \mathrm{dFS} 0712$ (G8), and 2dFS1829 (G2), provides further evidence for their supergiant luminosity class and thus membership to the SMC. The early-B-type stars appearing in these diagrams, in order of increasing magnitude at [24] are as follows: 2dFS0402, 2dFS2673 (both of type B0-5V; discussed above), AzV 9 (B0III), AzV 201 (B1(Be-Fe)), AzV 409 (or 2dFS2201; B0.5Ib), AzV 230 (B0Ib), and AzV 390 (B2Iab:). Of these, both AzV 409 and AzV 230 have spectral energy distributions (SEDs) with disk-like characteristics in all Spitzer passbands (i.e., similar to the Be star SEDs shown in Paper I, Figure 17), although the former has a slight upturn at [24]. The nature of these objects is discussed in Section 4.5. Finally, the late-B and AFG supergiants present in these diagrams are hypergiants whose spectra and SEDs indicate the presence of substantial stellar winds. A comparison of the colors of various types of massive stars in the LMC versus SMC CMDs reveals that the colors of the RSGs differ the most (see Section 4.5.3).

In Figures 6-8, we present TCDs using the near- and midinfrared photometry from our matched catalog. We label stars according to their spectral types and overplot all the SAGESMC detections in gray as Hess diagrams. Stars without dust have mid-infrared colors near 0 . Late-type stars can have $J-K_{s}$ excesses of $\leqslant 2 \mathrm{mag}$, while the group of stars with colors $K_{s}-[8.0] \sim 3,[4.5]-[8.0] \sim 2$, and [8.0] - [24] $3.5 \mathrm{mag}$ correspond to young stellar objects and background galaxies. We have overplotted six simple theoretical models to guide the

\footnotetext{
22 dFS3528 is discussed further in Section 4.5.2.
}

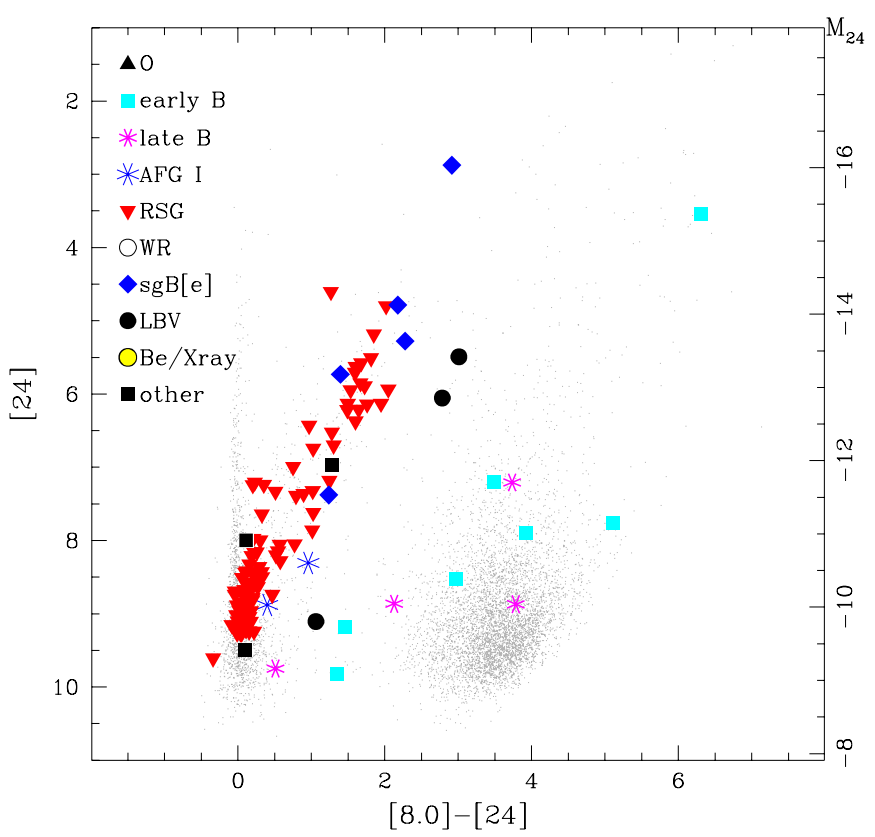

Figure 5. Same as Figure 4, but for the [24] vs. [8.0] - [24] color-magnitude diagram. The brightest sgB[e], RSGs, and LBVs are among the most luminous stars at $24 \mu \mathrm{m}$. The names and classifications of stars labeled as "other" are (in order of increasing [24] mag): 2dFS3528 (F0), 2dFS0712 (G8), and 2dFS1829 (G2).

(A color version of this figure is available in the online journal.)

interpretation of the stars in these diagrams, which are described in detail in Paper I: (1) a blackbody (BB) at various temperatures, (2) a power-law model $F_{v} \propto v^{\alpha}$, for $-1.5 \leqslant \alpha \leqslant 2$, (3) an OB star (represented by a 30,000 K BB) plus an ionized wind, (4) an $\mathrm{OB}$ star plus emission from an optically thin $\mathrm{H}$ II region, (5) an OB star plus $140 \mathrm{~K}$ dust, (6) a $3500 \mathrm{~K} \mathrm{BB}$ plus $250 \mathrm{~K}$ dust. The most conspicuous stars in all TCDs are the $\operatorname{sgB}[\mathrm{e}]$ stars, which have large excesses of $\sim 4 \mathrm{mag}$ in the $K-[8.0]$ color, similar to the $\mathrm{sgB}[\mathrm{e}]$ stars in the LMC. R4, a B0[e]-type $\mathrm{LBV}$, has mid-infrared colors intermediate between $\mathrm{sgB}[\mathrm{e}]$ and Be stars. We find a remarkable bimodal distribution of the OB stars, most clearly seen in Figure 6, with the redder grouping corresponding to $\mathrm{Oe}$ and Be stars (see Section 4.1 for more details). Among these outliers are the Be/X-ray binaries and other emission line stars, including some yellow supergiants. The near-infrared excesses found among the $\operatorname{sgB}[\mathrm{e}]$ and $\mathrm{W}-\mathrm{R}$ stars are on average lower than in the LMC, presumably due to the lower metallicity of the SMC. Finally, the RSGs, in contrast to their distribution in the LMC TCDs, are found to be clustered, due to the lower dust production rates at the metallicity of the SMC (see Section 5).

\section{INFRARED EXCESSES OF MASSIVE STARS IN THE SMC VERSUS THE LMC}

In this section, we study the infrared excesses of specific spectral types of massive stars, comparing them with the infrared excesses found in Paper I for the same types of stars in the LMC.

\subsection{O/Oe and Early-B/Be Stars}

In Figures 9 and 10, we plot $J_{\mathrm{IRSF}}$ versus $J_{\mathrm{IRSF}}-$ [3.6], $J_{\text {IRSF }}-[5.8]$, and $J_{\text {IRSF }}-$ [8.0] colors for the $208 \mathrm{O}$ and 1967 early-B stars in our catalog, respectively, denoting their luminosity classes, binarity, and emission line classification properties by different symbols. We compare the observed 


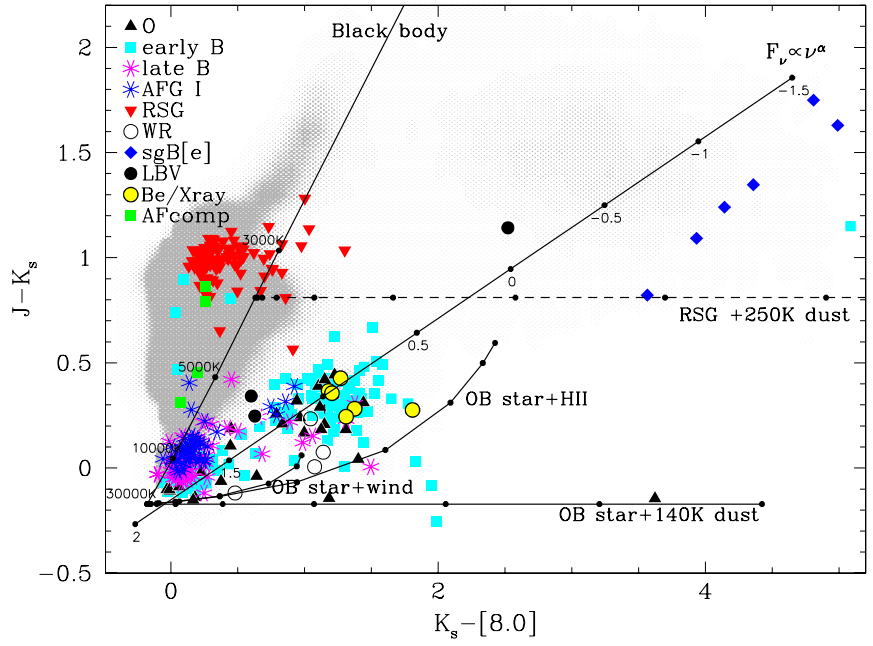

Figure 6. $J-K_{s}$ vs. $K_{s}-$ [8.0] diagram for massive stars in our catalog. The locations of all the SAGE detections are shown in gray as a Hess diagram. The solid lines represent models (described in Section 3): (1) a BB at various temperatures, as labeled, (2) a power-law model $F_{v} \propto v^{\alpha}$, for $-1.5 \leqslant \alpha \leqslant 2$, (3) an OB star plus an ionized wind, (4) an OB star plus emission from an optically thin H II region, (5) an OB star plus $140 \mathrm{~K}$ dust, (6) $3500 \mathrm{~K}$ BB plus $250 \mathrm{~K}$ dust (dashed line). The $\mathrm{sgB}[\mathrm{e}]$, RSGs, and the clump of "red" early-B stars that correspond to Be stars occupy distinct regions on this diagram.

(A color version of this figure is available in the online journal.)

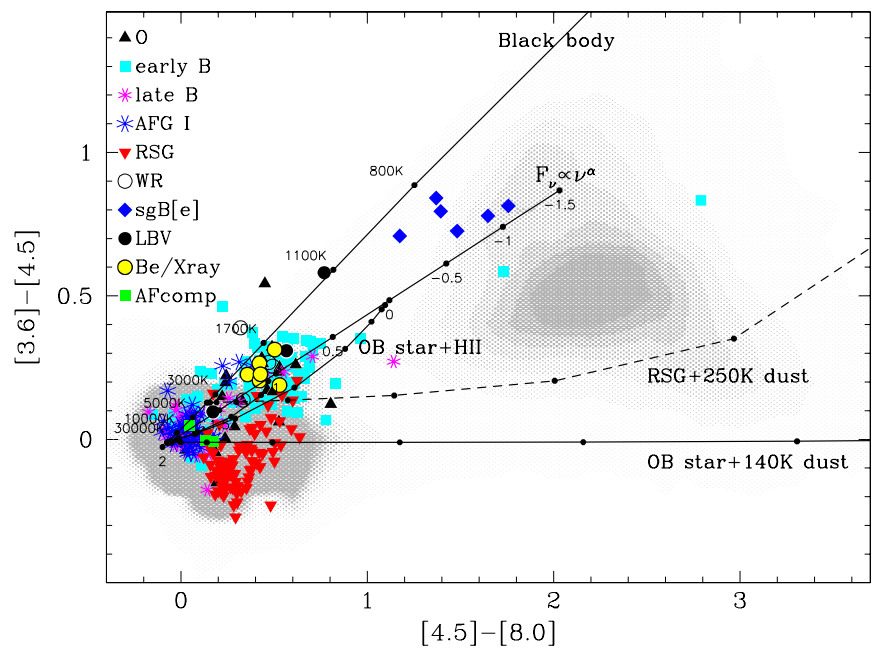

Figure 7. Same as Figure 6, but for the [3.6] - [4.5] vs. [4.5] - [8.0] diagram. The majority of hot massive stars lie between the BB and OB star +wind model, illustrating that a $\mathrm{BB}$ is a good approximation in the infrared.

(A color version of this figure is available in the online journal.)

colors with colors of plane-parallel non-LTE TLUSTY stellar atmosphere models (Lanz \& Hubeny 2003, 2007) of appropriate metallicity and effective temperatures. For reference, reddening vectors and TLUSTY models reddened by $E(B-V)=0.2 \mathrm{mag}$ are also shown. We clearly detect infrared excesses from free-free emission despite not having dereddened the stars, as in the LMC. At longer wavelengths, the excess is larger because the flux due to free-free emission for optically thin winds remains essentially constant with wavelength. Fewer stars are detected at longer wavelengths because of the decreasing sensitivity of Spitzer and the overall decline of their SEDs. We find that the majority of early-B supergiants in the SMC exhibit lower infrared excesses, when compared to their counterparts in the LMC (see Paper I), due to their lower mass-loss rates, although certain exceptions exist and deserve further study.

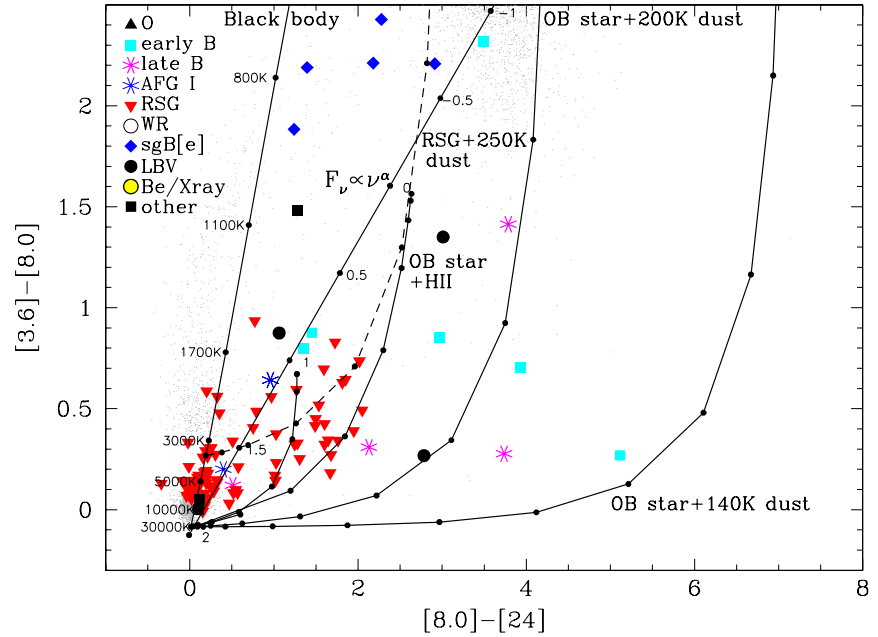

Figure 8. Same as Figure 6, but for the [3.6] - [8.0] vs. [8.0] - [24] diagram. The more uniform distribution of RSGs, as compared to the LMC, is due to the lower dust content.

(A color version of this figure is available in the online journal.)

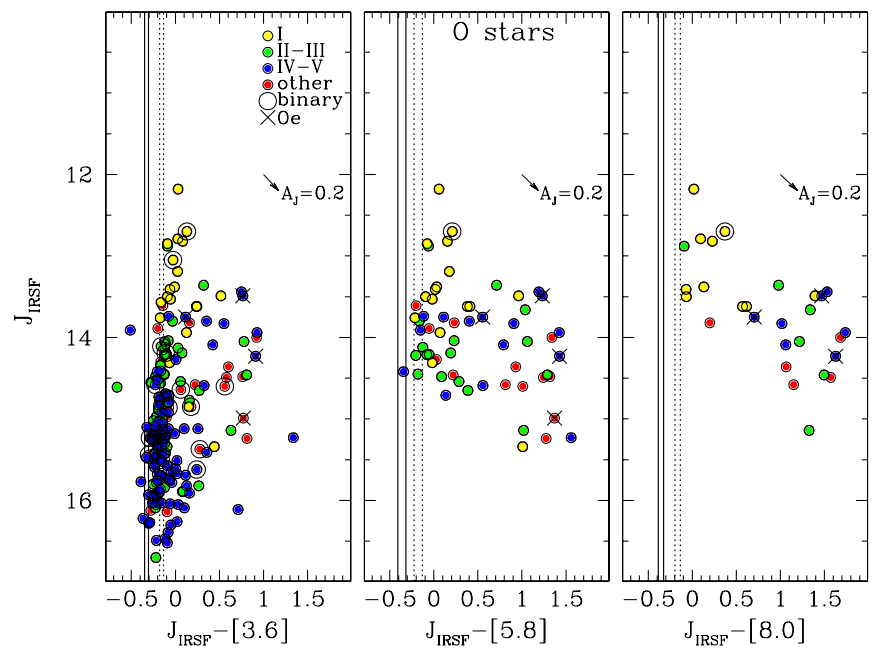

Figure 9. Infrared excesses ( $J_{\mathrm{IRSF}}$ vs. $J_{\mathrm{IRSF}}-[3.6], J_{\mathrm{IRSF}}-[5.8]$ and $\left.J_{\text {IRSF }}-[8.0]\right)$ for $208 \mathrm{O}$ stars. Supergiants are shown in yellow, giants in green, main-sequence stars in blue, stars with uncertain classifications ("other") in red, binaries with a large circle, and Oe stars with an $\times$. The solid lines correspond to $30 \mathrm{kK}$ and $50 \mathrm{kK}$ TLUSTY models with $\log g=4.0$. A reddening vector for $E(B-V)=0.2 \mathrm{mag}$ is shown, as well as reddened TLUSTY models by this same amount (dotted lines). The more luminous stars exhibit larger infrared excesses, which increase with $\lambda$.

(A color version of this figure is available in the online journal.)

The CMDs allow us to study the frequency of Oe and $\mathrm{Be}$ stars, given the low foreground and internal reddening for the SMC. Our SMC catalog contains four Oe stars among 208 O stars (see Figure 9), of which one is bluer than the rest. There are 16 additional stars with $J_{\text {IRSF }}-[3.6]>0.5 \mathrm{mag}$ and $J_{\text {IRSF }}<15 \mathrm{mag}$ (including all luminosity classes), whose spectra appear normal (although the $\mathrm{H} \alpha$ spectral region in most cases was not observed). We refer to these as "photometric Oe" stars and attribute their infrared excesses to free-free emission from a short-lived, possibly recurrent circumstellar region, whose $\mathrm{H} \alpha$ emission line was not detected during the spectroscopic observations either because the gas had dispersed or because the region was optically thick to $\mathrm{H} \alpha$ radiation or the observation spectral range just did not extend to $\mathrm{H} \alpha$. Given the expectation of lower $\dot{M}$ at SMC metallicity, we argue that 


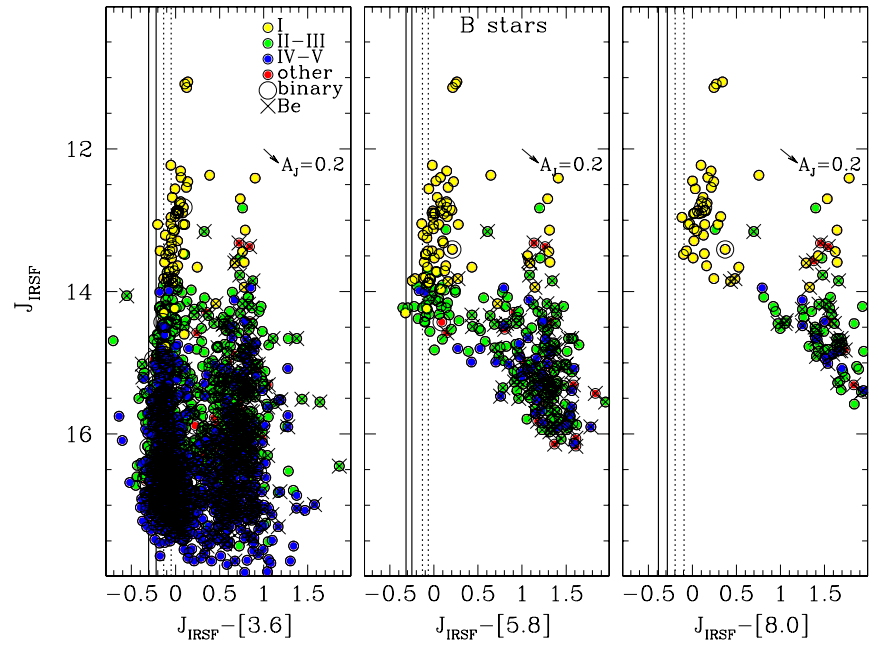

Figure 10. Same as Figure 9, but for 1967 early-B stars. The solid lines correspond to $20 \mathrm{kK}, \log g=3.0$ and $30 \mathrm{kK}, \log g=4.0$ TLUSTY models. A reddening vector for $E(B-V)=0.2 \mathrm{mag}$ is shown, as well as reddened TLUSTY models by this same amount (dotted lines). The distinct redder sequence, corresponding to the Be stars, implies the possibility of identifying or confirming Be stars photometrically.

(A color version of this figure is available in the online journal.)

such a region is more likely to be a transient disk rather than a wind. Assuming these are all Oe stars, we find a $10 \% \pm 2 \%$ fraction of Oe stars among the O stars in the SMC. The error in the fraction is dominated by small number statistics. In contrast, there are four Oe and 14 "photometric Oe" stars (with $J_{\text {IRSF }}-[3.6]>0.5 \mathrm{mag}$ and $J_{\text {IRSF }}<14.5 \mathrm{mag}$ ) out of $354 \mathrm{O}$ stars in the LMC (despite the higher $\dot{M}$ at LMC metallicity), which yields a $5 \% \pm 1 \%$ fraction of Oe stars among $\mathrm{O}$ stars in the LMC.

Turning to the early-B stars, the most striking feature in Figure 10 is a distinct sequence displaced by $\sim 0.8 \mathrm{mag}$ to the red. A large fraction of the stars falling on this redder sequence have Be star classifications, although not all Be stars reside there. Given that the circumstellar gas disks responsible for the emission in Be stars are known to completely vanish and reappear between spectra taken even one year apart (see review by Porter \& Rivinius 2003, and references therein), the double sequence reported here provides further evidence for the transient nature of the Be phenomenon. A bimodal distribution at the $L$ band was previously suggested by the study of Dougherty et al. (1994), which included a sample of 144 Galactic Be stars. Our larger Be sample, which is essentially unaffected by reddening, and the inclusion of all early-B stars, clearly confirms the bimodal distribution. It is due to the much larger number of Be stars classified in the SMC, in comparison to the LMC, as well as the higher fraction of Be stars among early-B stars in the $\mathrm{SMC}$, which is $19 \% \pm 1 \%$ versus $4 \% \pm 1 \%$ in the $\mathrm{LMC}$ when considering only the spectroscopically confirmed Be stars (cf. $\sim 17 \%$ for $<10$ Myr B0-5 stars; Wisniewski \& Bjorkman 2006). Excluding the targeted sample of Martayan et al. (2007a, 2007b) does not significantly bias the statistics, since the fraction only decreases to $15 \pm 1 \%$. We caution that incompleteness in our catalogs could also affect the determined fractions, if our sample turns out not to be representative of the whole population of $\mathrm{OB}$ stars.

We proceed to define "photometric Be" stars as early-B-type stars with an intrinsic color $J_{\text {IRSF }}-[3.6]>0.5 \mathrm{mag}$, given that a circumstellar disk or envelope is required to explain such large
Table 5

Oe and Be Star Fractions

\begin{tabular}{lrr}
\hline \hline \multicolumn{1}{c}{ Fraction } & LMC & \multicolumn{1}{c}{ SMC } \\
\hline Oe/O & $1 \% \pm 0.6 \%$ & $2 \% \pm 1 \%$ \\
$($ Oe+"phot Oe")/O & $5 \% \pm 1 \%$ & $10 \% \pm 2 \%$ \\
Be/early-B & $4 \% \pm 1 \%$ & $19 \% \pm 1 \%$ \\
(Be+"phot Be")/early-B & $16 \% \pm 2 \%$ & $27 \% \pm 2 \%$ \\
\hline
\end{tabular}

excesses. Including these "photometric Be" stars and using the same color and magnitude cuts as for the "photometric Oe" stars above, yields fractions of Be stars among early-B stars of $27 \% \pm 2 \%$ for the SMC and $16 \% \pm 2 \%$ for the LMC (cf. $32 \%$ from young SMC clusters; Wisniewski \& Bjorkman 2006). Table 5 summarizes these results. We compare our results with the fractions determined by Maeder et al. (1999) from young clusters, i.e., $39 \%$ for the SMC and $23 \%$ for the LMC, finding ours to be lower, although the sample selections were very different.

These preliminary statistics (available for the first time for Oe stars) indicate that both Oe and Be stars are twice as common in the SMC than in the LMC. We emphasize the importance of including the "photometric Be" stars, which significantly increase the frequencies of $\mathrm{Oe} / \mathrm{O}$ and $\mathrm{Be} /$ earlyB stars determined and are crucial when comparing such stars in different galaxies. This novel method of confirming Oe and Be star candidates from their infrared colors or a combination of their optical and infrared colors, as recently suggested by Ita et al. (2010a) is complementary to the detailed spectroscopic analyses by e.g., Negueruela et al. (2004) on individual Oe stars to understand their nature, although it is limited to galaxies with low internal reddening. We finally note that the spectral types of Oe stars in the SMC (O7.5Ve, O7Ve, O4-7Ve, and O9-B0III-Ve) and the LMC (O9Ve (Fe II), O7:Ve, O8-9IIIne, O3e) are earlier than those of known Galactic Oe stars, which are all found in the O9-B0 range (Negueruela et al. 2004).

To illustrate the double sequence more clearly, we present the same information in a histogram in Figure 11 for the early-B stars in both the LMC (from Paper I) and SMC, divided into magnitude bins. For stars with $J_{\text {IRSF }}-[3.6] \sim 0$, we find the brighter, more luminous stars to be redder (cf. top three panels of Figure 11), as in Paper I. In the second and third panels, the mean values of the $J_{\text {IRSF }}-[3.6]$ colors for the (blue, red) peaks are: $(-0.07,0.74)$ and $(-0.09,0.71) \mathrm{mag}$ for the SMC and $(-0.01$, $0.66)$ and $(-0.06,0.79) \mathrm{mag}$ for the LMC. The blue peak for the SMC stars is thus bluer than for the LMC, which is primarily due to the higher foreground reddening toward the LMC. In the second panel, we find the separation between peaks to be larger for the SMC (0.81 mag) than the LMC (0.67 mag), implying that the SMC Be stars have larger infrared excesses than their LMC counterparts. Unfortunately, in the third panel, the red peak of the LMC stars is barely discernible, therefore we cannot confirm this difference. We also ran a Kolmogorov-Smirnov test to check whether the stars are drawn from the same population (assuming the sample is unbiased). This yielded a probability of $5.0 \times 10^{-3}$ (for the stars in the second row) and $5.7 \times 10^{-5}$ (third row), implying that the underlying populations are indeed different.

Finally, we note that the brightest Be stars in the SMC $\left(J_{\text {IRSF }} \sim 13.2 \mathrm{mag}\right)$ are brighter than the brightest Be stars in the LMC ( $\left.J_{\text {IRSF }} \sim 13.4 \mathrm{mag}\right)$, i.e., there is a $0.7 \mathrm{mag}$ difference in absolute magnitude, given the 0.5 mag difference in the distance moduli. Garmany \& Humphreys (1985) were the first to make 


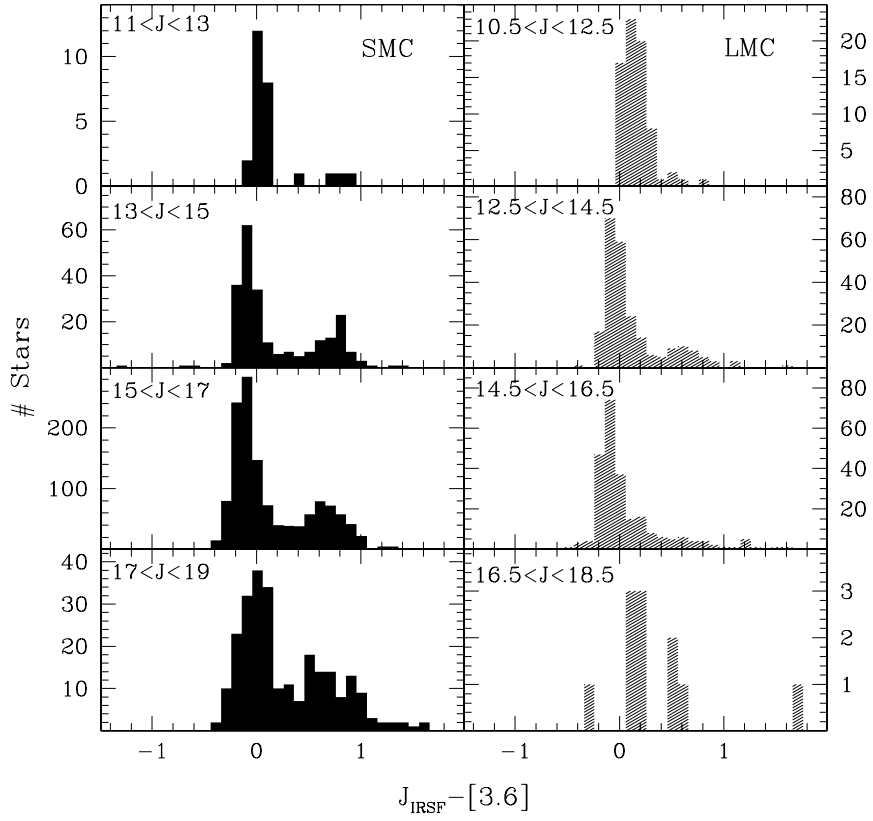

Figure 11. Histograms of the $J_{\text {IRSF }}-$ [3.6] colors of 1967 early-B stars in the SMC (left) and 586 in the LMC (from Paper I, right), divided into magnitude ranges. The main peaks of the histograms shift to redder colors for brighter stars. The redder secondary peaks correspond to the Be stars, which are more numerous in the SMC.

such a comparison, finding the Be stars in the Magellanic Clouds to be up to $1 \mathrm{mag}$ brighter when compared to Galactic Be stars, although their observation could be due to a selection effect, related to the selection of field versus cluster Be stars. Even though our sample is not dominated by such selection effects, we also find the brightest Be stars in the SMC to be intrinsically more luminous than their LMC counterparts. Given that winds are weaker at low metallicity, it is possible that there is a mechanism that allows disks to form at higher luminosities. ${ }^{23}$ Such a possibility would have interesting implications when extrapolating to even lower metallicities (see, e.g., Ekström et al. 2008).

\subsection{Be/X-ray Binaries}

Dray (2006) found that both metallicity and a star formation burst must be invoked to explain the large number of high-mass $\mathrm{X}$-ray binaries in the $\mathrm{SMC}$, and consequently the large number of Be/X-ray binaries. Of the $69 \mathrm{Be} / \mathrm{X}$-ray binaries in our SMC photometric catalog, 21 (or $30 \% \pm 8 \%$ ) were matched in the SAGE-SMC database (versus four matches out of $20 \mathrm{Be} / \mathrm{X}$-ray binaries or $20 \% \pm 11 \%$ in the LMC). Interestingly, some SMC $\mathrm{Be} / \mathrm{X}$-ray binaries are brighter than their LMC counterparts, despite being farther away, in agreement with the finding that $\mathrm{Be}$ stars in the SMC occur at higher luminosity. A comparison with the infrared colors of the Be/X-ray binaries in the LMC does not reveal any differences. The SEDs of the SMC Be/X-ray binaries show excess due to the disks around the Be stars, as in the LMC. Finally, the multiple $V I$ and $J H K_{s}$ measurements reveal variability for many systems, which deserves further study.

\subsection{Wolf-Rayet Stars}

Of the $12 \mathrm{~W}-\mathrm{R}$ stars in our spectral-type catalog, 10 were matched in the SAGE-SMC database. HD 5980 (SMC-WR5;

\footnotetext{
23 This would also explain the larger infrared excesses found in Be stars in the
} SMC, described in the previous paragraph.
AzV 229 WN6h; LBV binary) is labeled as an LBV in all diagrams and is the only W-R star with a detection at [24]. We compare the colors of the W-R stars to those of their LMC counterparts (see Paper I) and find that their infrared excesses are much lower, possibly indicating that their winds are much weaker than those of LMC W-R stars. Specifically, we find the SMC W-R stars to have "bluer" colors: $-0.2<J-[3.6]<0.5$ (versus $0.2-1.7$ for the LMC sample), $-0.2<J-K_{s}<0.1$ (versus 0-0.6), $0.4<K_{s}-[8.0]<1.2 \mathrm{mag}$ (versus 0.4-1.8). The origin of the hydrogen absorption lines seen in 10 of the $12 \mathrm{~W}-\mathrm{R}$ stars (classified as "WN+abs" or "WNha") remains unclear, since radial velocity surveys have only confirmed four as binary systems (Foellmi et al. 2003). The absorption in the other stars could alternatively be explained as photospheric absorption (due to weak winds; Massey et al. 2003). The infrared photometry made available for the W-R stars in this paper, will enable modeling of their SEDs to determine the properties of their stellar winds and perhaps the origin of the absorption lines.

\subsection{AF Composite Stars}

Evans et al. (2004a) discovered a class of objects with peculiar composite spectra (AF composites or AFcomp), which yield an A-type classification from their $\mathrm{Ca}$ II $\mathrm{K}$ lines, but an F-type classification from other metal lines and the $G$ band. For example, the classification AFA3kF8 denotes such an AF composite star, with Ca II K like A3 and $G$ band like F8. Our photometric catalog contains 16 out of the 20 AF composite stars from the $2 \mathrm{dF}$ Survey of Evans et al. (2004a). The AF composite stars that additionally exhibit forbidden emission lines (2dFS1804; AFA $3 \mathrm{kF0} / \mathrm{B}[\mathrm{e}]$ and 2dFS2837; AFA5kF0/B[e]) are labeled and henceforth treated as $\mathrm{sgB}[\mathrm{e}]$ stars.

The infrared colors of the AF composite stars provide further clues to their nature and confirm their astrophysical peculiarity. In Figure 2, their [3.6] - [4.5] colors are indistinguishable from AFG supergiants, ${ }^{24}$ while in Figure 3 they clearly have red colors, with $0.5<J-[3.6]<1$ mag. None are detected at [24]. In Figure 6, all four AF composite stars with [8.0] detections show near-infrared colors in the range $0.3<J-K_{s}<0.9$ mag; thus, intermediate between blue and red supergiants, possibly indicating these stars are on blue loops or in interacting binaries. The SEDs indeed are quite "flat;" thus, not consistent with normal single stars, while the multiple $V I$ and $J H K_{s}$ measurements reveal variability for several sources, which in some cases can be confirmed by light curves available from the OGLE database (Udalski et al. 1997; Szymański 2005). We note that $2 \mathrm{dFS} 2945$ (HV 11519) is a known $\delta$ Cep pulsator.

\subsection{Dusty Stars}

Our catalog includes 166 stars with detections at [24], which indicate the presence of dust. Of these, 97 are RSGs, five $\mathrm{sgB}[\mathrm{e}]$, and three LBV stars, all exhibiting dust, as expected. Luminous supergiants with strong winds or disk-like SEDs also exhibit infrared excess at [24] and [8.0] and therefore appear in Figures 4 and 5. There are four B0-5 V type stars: 2dFS0402, 2dFS2411, 2dFS2673, and 2dFS3701 that have very flat SEDs. Additionally, we find a class of 44 dusty OB stars (16 late-O and 28 early-B stars, making up $2 \%$ of our sample), with similar characteristics to the peculiar population of dusty OB stars reported by Bolatto et al. (2007, 190 O9-B3 stars, corresponding to $5 \%$ of their sample) in their $\mathrm{S}^{3} \mathrm{MC}$ survey and

\footnotetext{
${ }^{24}$ The brightest AF composite star at [3.6] is 2dFS5049.
} 


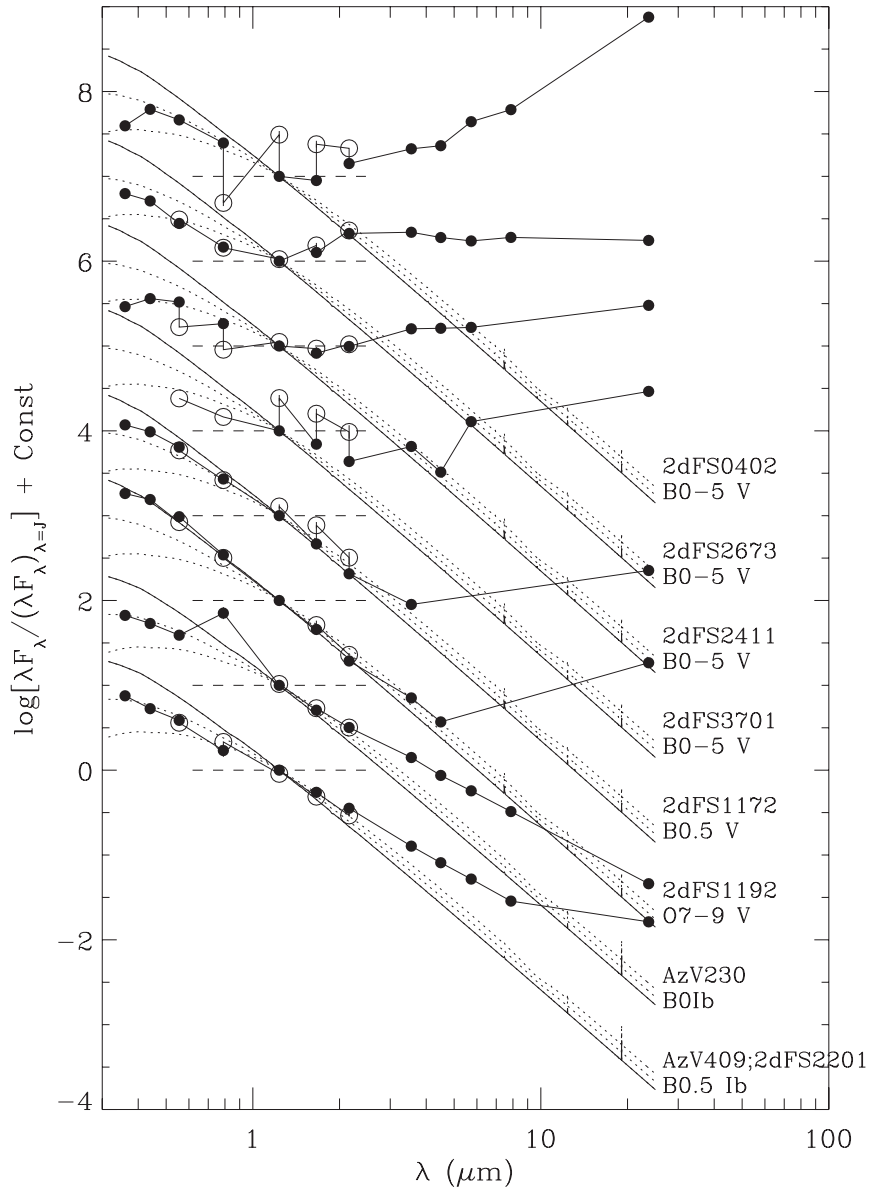

Figure 12. SEDs of OB stars with [24] detections, normalized by their $J$-band fluxes (dashed line) and offset for display purposes. Normalized TLUSTY model atmospheres at SMC metallicity $(30 \mathrm{kK}, \log g=3.00$ for the main-sequence stars; $20 \mathrm{kK}, \log g=2.25$ for the two supergiants) are overplotted as solid lines for comparison. The MCPS, IRSF, and SAGE measurements (filled circles) and the 2MASS and OGLE measurements (open circles) are also connected by a solid line. The dotted curves correspond to TLUSTY models reddened by $E(B-V)=0.25$ and $0.50 \mathrm{mag}$. Several classes of object are represented (see Section 4.5). 2dFS1172 and 2dFS1192 are examples of dusty OB stars. Variability is present in several sources; however, in the case of 2dFS0402 it is likely due to a mismatch with a close infrared source.

recently confirmed by Ita et al. (2010b) with data from AKARI. Hereafter, we refer to these stars as "dusty OB stars." They are characterized by a photospheric SED out to [5.8] and strong [24] excess, which cannot be attributed to free-free emission. Most have main-sequence luminosity classes, although there are a few giants and supergiants. In Figure 12, we plot SEDs for the four stars with unusually flat or rising SEDs, two representative examples from among the 44 "dusty OB stars" (2dFS1172, 2dFS1192) and two examples of luminous supergiants with infrared excess due to strong winds or disks (AzV 230, AzV 409; 2dFS2201). For completeness, in Figure 13, we show two examples of late-B supergiants with [24] detections and the five AFG supergiants that have [24] detections. Note, the F0 supergiants R47 (a rare eclipsing binary; Prieto et al. 2008) and 2dFS3528 (an LBV candidate, see Section 4.5.2) are of particular interest.

The dust detected in the class of "dusty OB stars" could either be directly related to the OB stars or simply be contamination given the volume of the SMC sampled with the 6" resolution of MIPS. While inspection of their environments shows that they are located in or near young associations, they are frequently not

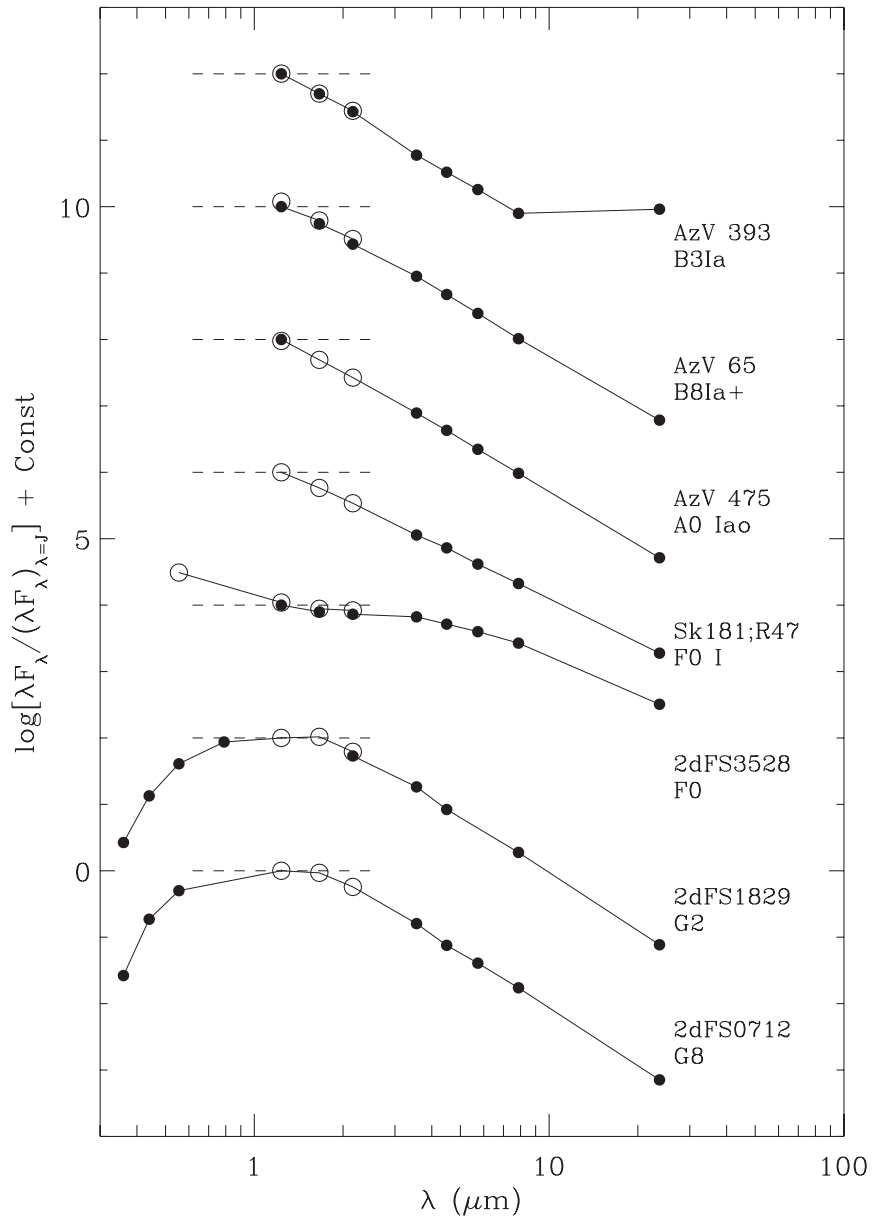

Figure 13. SEDs of two late-B supergiants and of the five AFG supergiants detected at [24], normalized by their $J$-band fluxes (dashed line) and offset for display purposes, as in Figure 12. The SED of the F supergiant 2dFS3528 most resembles that of R4 (AzV 16, B0[e]LBV); we suggest it to be an LBV candidate.

the youngest objects (earliest types), e.g., NGC 330 has three "dusty OB stars" nearby and a turn-off of around B2 (Lennon et al. 1993). We examined the spectra for 18 of these sources (12 from the 2dF Survey and six from the VLT-FLAMES Survey; Evans et al. 2006), which all exhibit sharp nebular-like Balmer emission lines; for those with red-optical spectra, seven also have $\mathrm{H} \alpha$ nebular emission and three have weak [N II] and [S II] lines, indicating the presence of a faint $\mathrm{H}$ II region. Many sources for which we do not have spectra available are either listed in $\mathrm{H} \alpha$ emission line catalogs or lie in regions of faint nebulosity. Given that most "dusty OB stars" are not found within very young regions, we suggest that these sources represent unrelated cirrus hot spots or cases where hot stars are ionizing nearby dusty molecular clouds (rather than young stellar objects) producing both the nebular emission lines and emission from warm dust (as in Cepheus B, see e.g., Panagia \& Thum 1981).

\subsubsection{Supergiant B[e] Stars}

In the SMC photometric catalog, we have detected seven luminous sources with colors typical of $\mathrm{sgB}[\mathrm{e}]$ stars (see Paper I for an introduction), i.e., $M_{3.6}<-8$, [3.6] $-[4.5]>0.7$, and $J-[3.6]>2 \mathrm{mag}$ (see Figures 2 and 3). Five of these are previously known $\mathrm{sgB}[\mathrm{e}]$ stars (with R50; B2-3[e] being the brightest in all IRAC and MIPS bands), while R4 (AzV 16) is classified as an LBV with a $\operatorname{sgB}[\mathrm{e}]$ spectral type. In 


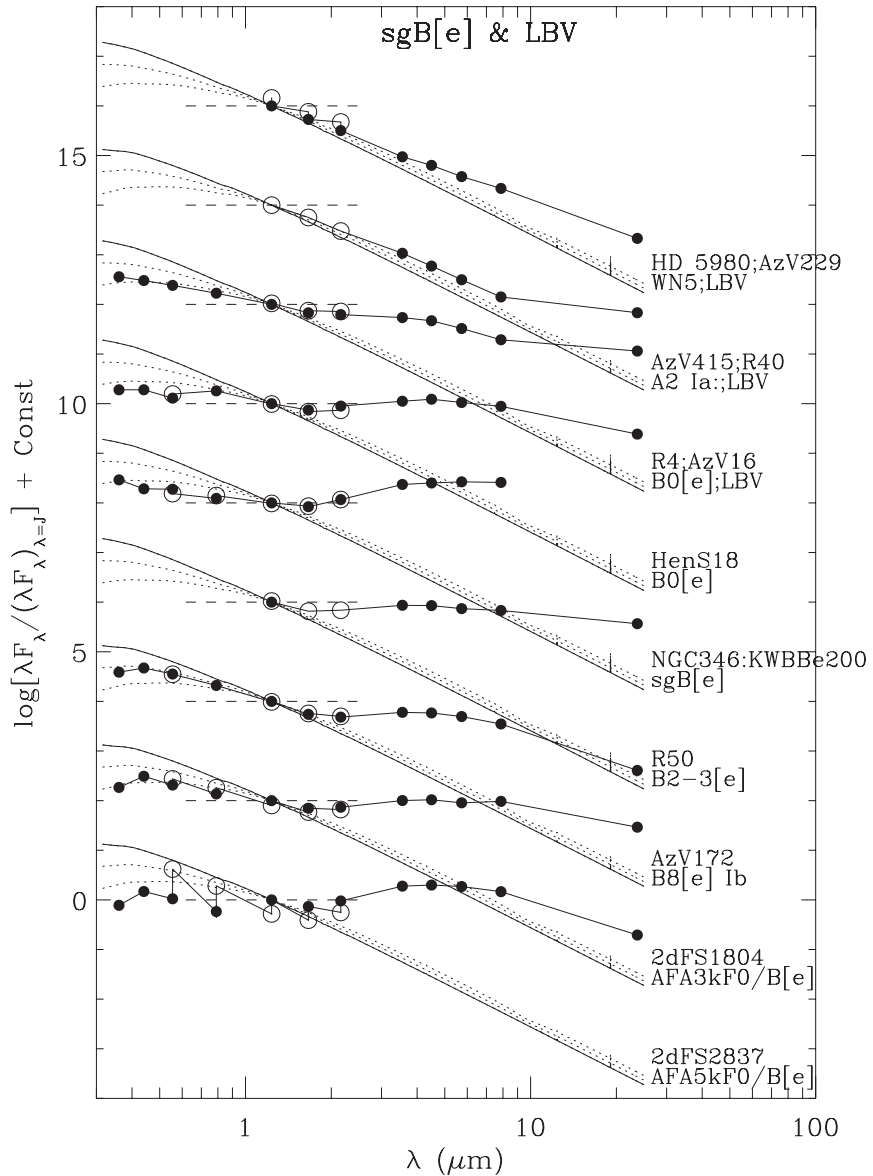

Figure 14. SEDs of the LBVs and $\mathrm{sgB}[\mathrm{e}]$, all detected at [24], normalized by their $J$-band fluxes (dashed line) and offset for display purposes, as in Figure 12. TLUSTY model atmospheres are overplotted for comparison: $15 \mathrm{kK}, \log g=1.75$ for the B8 and later types; $20 \mathrm{kK}, \log g=2.25$ for the earlier types. The last two SEDs with composite spectral types closely resemble the $\mathrm{sgB}[\mathrm{e}] \mathrm{SEDs}$; note, these stars vary.

addition to these, we find that 2dFS1804 (AFA3kF0/B[e]) has a very similar SED (and therefore infrared colors) to the known sgB[e] 2dFS2837 (AFA5kF0/B[e]). Evans et al. (2004a) also remarked on the similarity of their spectra. We therefore confirm the supergiant nature of $2 \mathrm{dFS} 1804$ and present its SED in Figure 14 along with those of other $\operatorname{sgB}[\mathrm{e}]$ and LBV stars. We note that both are photometrically variable. The similarity of the SEDs of these $\mathrm{sgB}[\mathrm{e}]$ stars, despite the various optical spectral classifications, implies that all are the same class of object. The cooler, composite spectral types indicate a lower mass and perhaps a transitional stage to or from the $\operatorname{sgB}[\mathrm{e}]$ phenomenon. The only difference we find between the $\operatorname{sgB}[\mathrm{e}]$ stars in the SMC versus the LMC is that on average they are 1-2 mag fainter (in absolute terms).

\subsubsection{Luminous Blue Variables}

All three known LBVs in the SMC: R4 (AzV 16, B0[e]LBV), R40 (AzV 415, A2Ia: LBV), and HD 5980 (WN6h;LBV binary), were all detected at infrared wavelengths. In all CMDs and TCDs, R4 is the more reddened LBV. It occupies a location similar to other $\mathrm{sgB}[\mathrm{e}]$ stars on the CMDs; however, in Figures 6 and 7 it lies between the $\operatorname{sgB}[\mathrm{e}]$ and the Be stars, whereas the colors of HD 5980 (a well-known eccentric eclipsing binary, see e.g., Foellmi et al. 2008) are similar to those of the LBVs in the LMC. We plot their SEDs in Figure 14 and find them to differ, given their very different spectral types. Moreover, we find evidence for variability, which can be confirmed from existing light curves in the All Sky Automated Survey (ASAS; Pojmański 2002), as pointed out by Szczygiel et al. (2010), who studied the variability of the massive stars presented in Paper I in the LMC. The various SED shapes and spectral types observed depend on the time since the last outburst event and the amount of dust formed.

Finally, we note that the F0 star, 2dFS3528 (Sk166) also lies among the $\operatorname{sgB}[\mathrm{e}]$ stars in Figures 4, 5, and 8 and near R4 (AzV 16; B0[e]LBV) in the other CMDs and TCDs, because it has a similar SED (cf. Figures 13 and 14). Although Sanduleak originally classified this star as "OB:," its $2 \mathrm{dF}$ spectrum is clearly F-type, but with strong $\mathrm{H} \alpha$ emission having broad wings, which indicate a wind. Osmer (1973) designated it as a nonmember based on its optical colors. It is included in the catalog of Massey $(2002)^{25}$ and indeed has some colors that are too blue, while others are too red for its spectral type. However, the radial velocity measured by Evans \& Howarth $\left(2008,+137 \pm 8 \mathrm{~km} \mathrm{~s}^{-1}\right)$ firmly places it in the SMC. The SED of 2dFS3528 is distinctly $\operatorname{sgB}[\mathrm{e}]-$ like and most similar to that of R4. All the above characteristics, along with the variability detected in the nearinfrared, make this star an LBV candidate. Further monitoring is highly desirable to confirm its LBV nature.

\subsubsection{Red Supergiants}

The RSGs are among the brightest mid-infrared sources in the SMC, with absolute magnitudes reaching $-12 \mathrm{mag}$ at [3.6], and -13 mag at [8.0] and [24]. However, in the LMC, some RSGs occur at higher [24] luminosities. SMC_018592 (K0-2I) is the brightest RSG at [3.6], [8.0], and [24] (see Figures 2, 4, 5). In Figure 3, the following three RSGs have "bluer" colors than the rest (in order of increasing [3.6] magnitude): SMC_030135 (K2I), SMC_035231 (K2I), and SMC_049990; 2dFS1517. The latter has two classifications: K5I by Massey \& Olsen (2003) and G2 by Evans et al. (2004a), making it a variable candidate (also given its radial velocity: $+143 \pm 5 \mathrm{~km} \mathrm{~s}^{-1}$; Evans \& Howarth 2008). In Figure 6, SMC_035231 (K2I) is the outlier at $(0.4,0.6)$, making it a possible foreground dwarf; while SMC_058738 (K2I) is the outlier at $(1.0,0.5)$. We note that SMC_055188 (M3-M4I) and SMC_046662 (M0I) were reported by Levesque et al. (2007) to have variable spectral types; they are included in our catalog and are among the more reddened RSGs.

A comparison of the SMC CMDs and TCDs to the corresponding ones of the LMC from Paper I reveals that the distribution of RSG colors is quite different. The SMC RSGs have a dominant population of non-dusty RSGs and a population of dusty RSGs, with a distinct color distribution. In contrast, almost all RSGs in the LMC are dusty to some degree, with a rather continuous distribution in color. This difference is due to the lower dust content $(\propto Z)$, which is not compensated for by the lower wind velocity ( $\propto \sqrt{Z}$, see van Loon 2000, 2006; van Loon et al. 2008). Furthermore, since both the dust production rate (cf. Sloan et al. 2008) and the dust-to-gas ratio depend on $Z$ (van Loon 2006), $\dot{M}$ is independent of $Z$, though the wind velocities of RSGs depend on metallicity (see, e.g., Habing et al. 1994; Marshall et al. 2004). While the optical and near-infrared colors of RSGs have long been known to display a $Z$ dependence (cf. Arp 1959; Oestreicher et al. 1997), the systematics of

\footnotetext{
${ }^{25}$ [M2002] 80339; $V=12.92, B-V=0.15, U-B=-0.45$, and $V-R=0.17 \mathrm{mag}$
} 


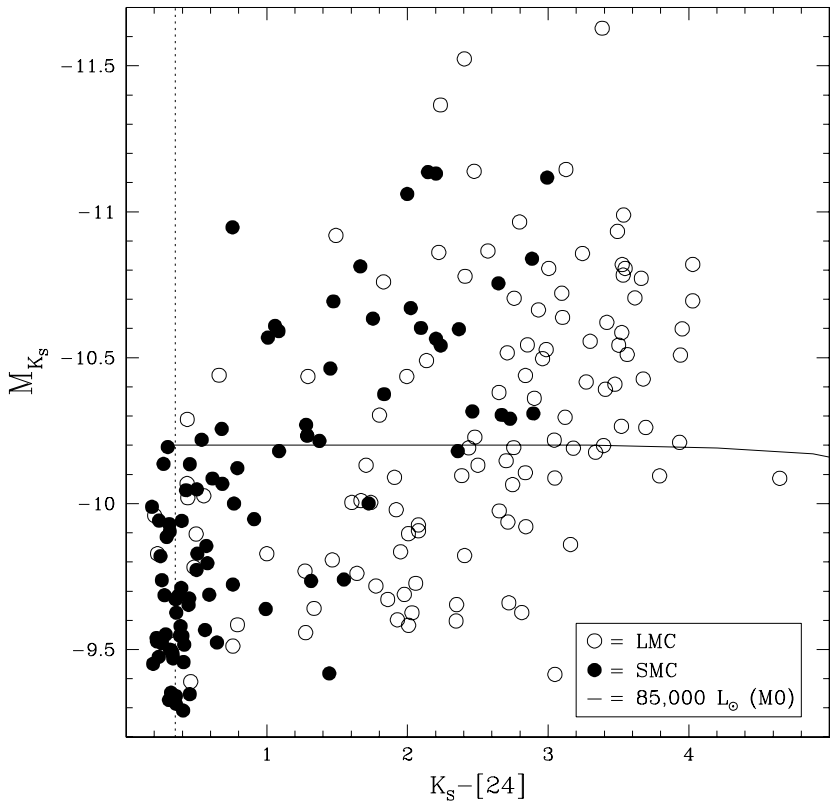

Figure 15. $M_{\mathrm{K}_{\mathrm{s}}}$ vs. $K_{s}-$ [24] CMD of our sample of RSGs in the SMC (filled circles; this work) and LMC (open circles; Paper I). The fainter SMC RSGs cluster around $K_{s}-[24]=0.35$ (dotted line). A model from Groenewegen (2006) for a star with spectral type M0 and luminosity $85,000 L_{\odot}$ with increasing amounts of dust toward redder color is shown for reference. Most LMC stars and many SMC stars show clear excess emission due to dust, which is most pronounced at high luminosity.

their mid-infrared colors have only been investigated for limited samples (e.g., Groenewegen et al. 2009).

\section{RED SUPERGIANT MASS-LOSS RATES}

Our large samples of RSGs in the SMC and LMC lend themselves to a study of the $Z$ dependence of the mass-loss rate. Figure 15 presents the $M_{K_{s}}$ versus $K_{s}-$ [24] CMD for both SMC and LMC RSGs (from Paper I), on which the RSGs are spread out as a result of various amounts of circumstellar dust. The fainter RSGs in the SMC concentrate around a vertical sequence at $K_{s}-[24] \approx 0.35 \mathrm{mag}$, but all other RSGs have significantly redder $K_{s}-$ [24] colors indicative of the emission from dust. A series of models for a star with spectral-type M0 and luminosity 85,000 $L_{\odot}$ (Groenewegen 2006) are plotted for reference, showing that the dust remains optically thin at nearinfrared wavelengths for $K_{s}-$ [24] $<5$ mag.

The $K_{s}-$ [24] color is a good measure of the optical depth of the circumstellar dust envelope. We use the results from radiative transfer modeling presented in Groenewegen (2006) for a star of luminosity $L=3000 L_{\odot}$, wind speed $v=10 \mathrm{~km} \mathrm{~s}^{-1}$, dust-togas ratio $\psi=0.005$, surrounded by silicate grains with a size of $0.1 \mu \mathrm{m}$ and a condensation temperature of $1000 \mathrm{~K}$. The relation between this reference $\dot{M}$ and $K_{s}-$ [24] color is shown in Figure 16, for a star of spectral-type M0 (typical for the SMC stars) and again for a star of spectral-type M6 (at the cool extreme of the LMC stars in our sample). For colors $K_{s}-[24]<$ $0.8 \mathrm{mag}$, the relation is rather steep; given the inaccuracies in photometry and stellar parameters we will not assign mass-loss rates to these non-dusty stars. For colors $0.8<K_{s}-[24]<5$ mag, the relation is quite well approximated by the formula:

$$
\log \left(\dot{M}_{\text {reference }}\right)=-8.6+0.5 \times\left(K_{s}-[24]\right) .
$$

The deviations from this formula are not (much) more than a factor of 2 for individual stars and will tend to average out when considering the sample as a whole.

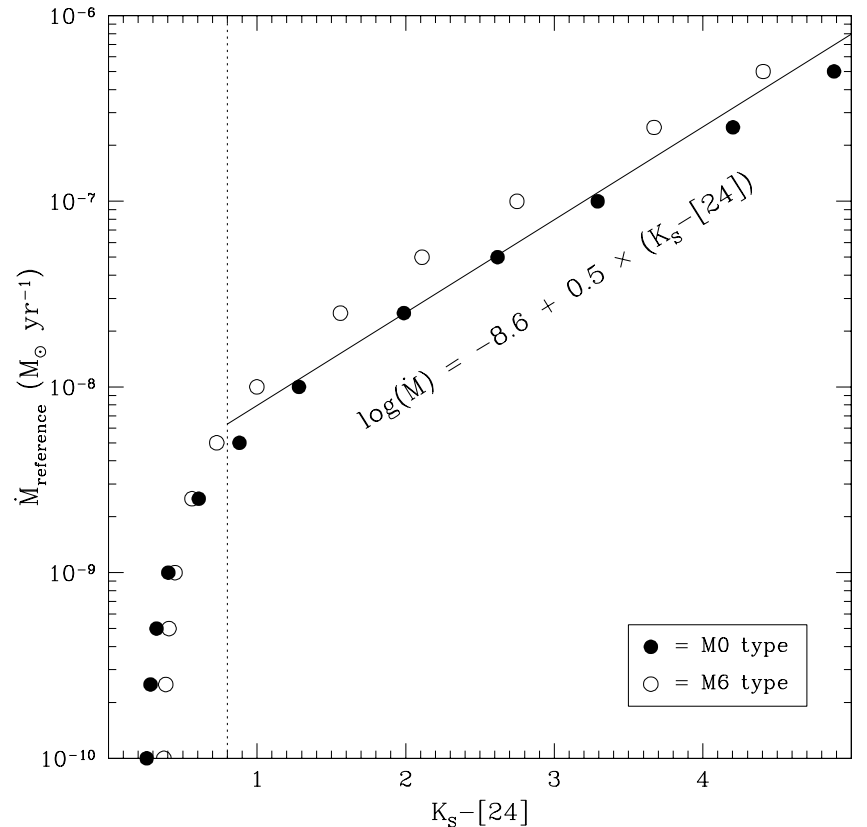

Figure 16. $\dot{M}$ as a function of $K_{s}-$ [24] for two sets of models from Groenewegen (2006) with spectral types M0 (filled circles) and M6 (open circles). The solid line indicates the approximation described by the labeled equation, which is valid for a star of luminosity $3000 L_{\odot}$, wind speed $10 \mathrm{~km} \mathrm{~s}^{-1}$ and dust-to-gas ratio 0.005 , for the range $0.8<K_{s}-[24]<5$ mag. We use scaling relations to apply it to the RSGs in the Magellanic Clouds.

The true $\dot{M}$ is obtained by applying scaling relations valid for dust-driven winds (cf. van Loon 2007):

$$
\dot{M}=\dot{M}_{\text {reference }}(v / 10)(0.005 / \psi)(L / 3000)^{0.5},
$$

where the wind speed scales as (e.g., Marshall et al. 2004)

$$
v=10(\psi / 0.005)^{0.5}(L / 3000)^{0.25} .
$$

For the dust-to-gas ratio $\psi$, we adopt a value roughly in proportion to the metallicity of the stellar population, namely, $\psi \approx 0.001$ for the SMC and $\psi \approx 0.002$ for the LMC (cf. van Loon 2000, 2006). The luminosity is obtained by scaling the $K_{s}$-band magnitude of the model star from Groenewegen (2006); for the SMC stars we adopt $K_{s}=8.1 \mathrm{mag}$ for the $3000 L_{\odot}$ star, at a reference distance of $8.5 \mathrm{kpc}$ (this corresponds to a spectral-type M0) and $K_{s}=7.9 \mathrm{mag}$ for the LMC stars (spectral type about M2). The distance modulus to the SMC is taken as $18.91 \mathrm{mag}$ and to the $\mathrm{LMC}$ as $18.41 \mathrm{mag}$ (to be consistent with Paper I). The inaccuracies in the luminosities resulting from this approximation are of the order of $20 \%$, which is small for our purposes.

The estimated mass-loss rates show a clear positive correlation with luminosity (see Figure 17), confirming previous results (van Loon et al. 1999, 2005; van Loon 2006; Groenewegen et al. 2009) with a larger sample of stars. Apparently, the fainter RSGs are not very proficient in their mass loss. However, these massloss rates were derived by assuming that dust condenses in these winds at the efficiencies expected from their metal content. This may not be true, particularly in the relatively warm atmospheres of early-M or late-K type stars, where the dust-to-gas ratio can be much less than assumed here (cf. van Loon et al. 2005). Indeed, at $L<9 \times 10^{4} L_{\odot}$ most of the RSGs in the SMC show very little evidence of dust at all, but they may still exhibit a purely gaseous wind. On the other hand, the values for the wind speed are estimated to be $9-12 \mathrm{~km} \mathrm{~s}^{-1}$ for the SMC sample 


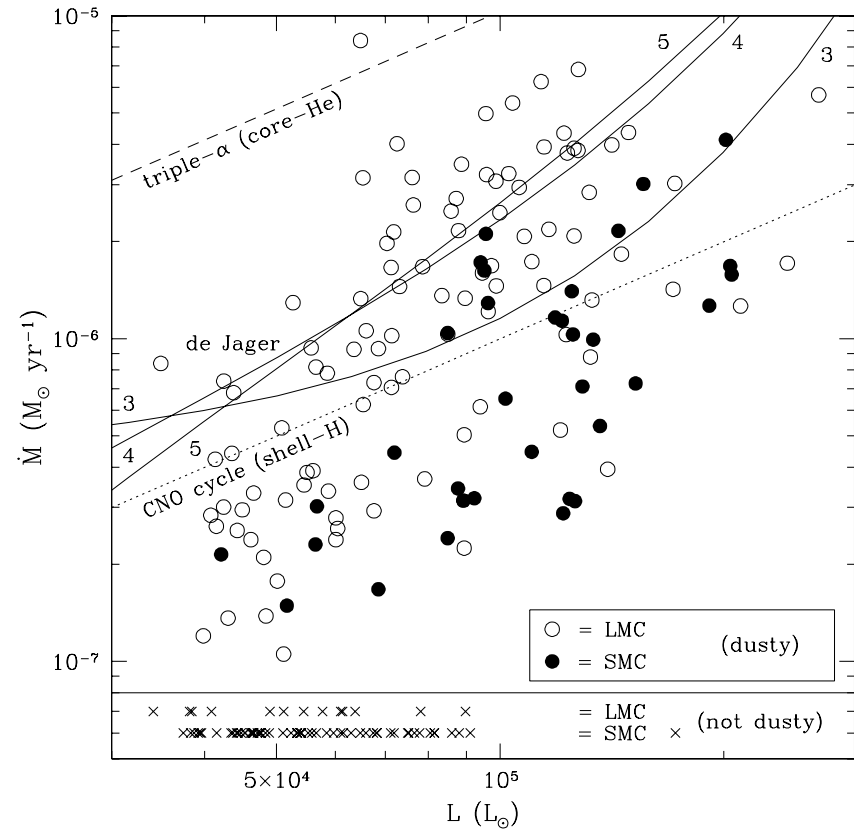

Figure 17. $\dot{M}$ vs. luminosity $L$ for our samples of RSGs in the SMC (filled circles; this work) and LMC (open circles; Paper I). No mass-loss rates could be determined for stars with $K_{s}-[24]<0.8 \mathrm{mag}$; these are shown in the bottom panel at their respective luminosities (crosses). Shown for reference are the nuclear burning rates for the triple- $\alpha$ process at work in helium burning in the cores of RSGs (dashed line), as well as the CNO cycle in hydrogen shell burning (dotted line) and the prescription of de Jager et al. (1988) for 3000, 4000 , and $5000 \mathrm{~K}$ (solid lines, labeled by the first digit of the temperature).

(12-19 $\mathrm{km} \mathrm{s}^{-1}$ for the LMC sample). This is consistent with expectations for a fluid in which the grains are well coupled to the gas and thus can transfer momentum from the radiation field onto the bulk matter (cf. Marshall et al. 2004); much lower values could bring this assumption into question (cf. McDonald et al. 2009).

The mass-loss rate is next compared to the rate at which matter is converted into energy in the central engine of the star, to assess whether much mass is lost before the star is taken elsewhere on its evolutionary path. In the context of core-helium burning RSGs, the most relevant process is the triple- $\alpha$ process, which has an efficiency $\eta \approx 5.9 \times 10^{17} \mathrm{erg} \mathrm{g}^{-1}$ (Kippenhahn $\&$ Weigert 1990); we also compare to the CNO cycle, which governs hydrogen shell burning and has $\eta \approx 6.1 \times 10^{18} \mathrm{erg} \mathrm{g}^{-1}$. The nuclear burning rate (in $M_{\odot} \mathrm{yr}^{-1}$ ) is then

$$
\dot{M}_{\text {nuclear }}=6.087 \times 10^{7}(L / \eta)
$$

We find that essentially all RSGs in our samples lose mass through stellar winds at a significantly lower rate than that at which helium is burned in their cores (Figure 17), often by an order of magnitude difference. This is in stark contrast to samples of RSGs that were selected from among the brightest mid-infrared sources; van Loon et al. (1999) showed that most of those RSGs have mass-loss rates similar to the nuclear burning rate, with a few extreme objects exceeding that rate by a significant amount. The optically selected RSGs in the SMC and LMC are therefore found to be in a more stable phase of RSG evolution, in contrast to the RSGs selected as bright mid-infrared sources, which have a shorter lifetime. The prescriptions of de Jager et al. (1988) for 3000, 4000, and $5000 \mathrm{~K}$ coincide with the most luminous LMC RSGs, but are too high for the less luminous RSGs (in both galaxies). The $3000 \mathrm{~K}$ prescription of de Jager et al. (1988) better reproduces the luminous RSGs in the SMC, whereas the warmer prescriptions better reproduce the luminous RSGs in the LMC. This is in conflict with the observed differences in temperature, with the SMC RSGs having earlier spectral types than their LMC counterparts.

The SMC sample on the whole exhibits lower dusty massloss rates than the LMC sample (Figure 17), and RSGs in the SMC appear to contribute relatively more at higher luminosities $\left(L>9 \times 10^{4} L_{\odot}\right)$, while RSGs in the LMC also contribute noticeably at fainter luminosities. Both observations are likely related to the warmer atmospheres (earlier spectral types) of the SMC RSGs compared to those in the LMC. We thus conjecture that dusty mass loss plays a less important role in the SMC than in the LMC, while other forms of mass loss may instead be more important. For instance, as recently suggested (van Loon et al. 2010a), the warmer winds of metal-poor RSGs could be more susceptible to the coupling of Alfvén waves, which could provide a driving mechanism in the absence of radiation pressure.

We emphasize that our samples miss the dustiest RSGs, which are in brief episodes of very high mass loss; therefore, our results only apply to the optically bright RSG phase. A few such very dusty RSGs (known to exist in the Magellanic Clouds, e.g., Wood et al. 1992; Groenewegen \& Blommaert 1998; van Loon et al. 1999, 2010a, 2010b; Srinivasan et al. 2009) contribute more dust than the entire samples of optically bright RSGs considered here.

\section{SUMMARY}

This paper presents the first catalogs of accurate spectral types and multi-wavelength photometry of massive stars in the SMC, which are used to study their infrared properties. The spectroscopic catalog contains 5324 massive stars, with accurate positions and spectral types compiled from the literature, and constitutes the largest such catalog currently available for any galaxy. The photometric catalog comprises uniform 0.3-24 $\mu \mathrm{m}$ photometry in the $U B V I J H K_{s}+$ IRAC+MIPS24 bands for a subset of 3654 stars that were matched in the SAGE-SMC database. The low foreground reddening toward the SMC, and the identical distance of the stars minimize systematic errors due to reddening and enable the investigation of infrared excesses. As in Paper I, we construct CMDs and TCDs, and discuss the position of $\mathrm{O}$, early- and late-B stars, W-R, LBV, sgB[e], classical Be stars, RSGs, AFG supergiants, and $\mathrm{Be} / \mathrm{X}$-ray binaries on them. These diagrams are useful for interpreting infrared photometry of resolved massive stars in nearby galaxies at low metallicity.

A comparison of the infrared colors of the massive stars in the SMC to those of their counterparts in the LMC (presented in Paper I) reveals differences that are due to the different evolution at SMC metallicity. The main results of our study concern the emission line Oe and Be stars, and the RSGs. We detected a clear bimodal distribution of early-B stars, with the redder sequence corresponding to classical Be stars and therefore propose that Be stars (and similarly Oe stars) can be easily discriminated photometrically, by their infrared colors. We find the fraction of emission line stars in the SMC $(10 \% \pm 2 \%$ for Oe and $27 \% \pm$ $2 \%$ for $\mathrm{Be})$ to be double that of the LMC $(5 \% \pm 1 \%$ for $\mathrm{Oe}$ and $16 \% \pm 2 \%$ for $\mathrm{Be}$ ), when including the "photometric" Oe and $\mathrm{Be}$ stars. This is the first time the frequency of Oe stars is determined beyond the Galaxy and at subsolar metallicities. We also find Be stars and Be/X-ray binaries to occur at higher luminosity, $\operatorname{sgB}[\mathrm{e}]$ stars to be on average less luminous than their counterparts in the LMC (at [3.6]), and W-R stars to have 
smaller excess, all due to the different evolution at the lower metallicity of the SMC.

The infrared colors of the RSGs in the SMC are found in most cases to be consistent with little dust, with only the most luminous sources showing excess emission presumably from circumstellar dust. This is in contrast to the generally dusty RSGs in the LMC and agrees with the expectation that the dust content in metal-poor RSGs is lower. We find that the mass-loss rates in SMC RSGs correlate positively with luminosity, as in the LMC. Finally, we confirm the astrophysical peculiarity of the composite A \& F type spectra discovered by Evans et al. (2004a), the $\mathrm{sgB}[\mathrm{e}]$ nature of $2 \mathrm{dFS} 1804$, and find the F0 supergiant $2 \mathrm{dFS} 3528$ to be an LBV candidate. This paper thus demonstrates the wealth of information contained in the SAGE-SMC survey and enables studies of the infrared properties of massive stars as a function of metallicity, in combination with the SAGE survey of the LMC. Studies of particular classes of massive stars and whole massive star populations in nearby galaxies, as outlined in Paper I, are obvious directions for follow-up.

A.Z.B. acknowledges support from the Riccardo Giacconi Fellowship award of the Space Telescope Science Institute and from the European Commission Framework Program Seven under a Marie Curie International Reintegration Grant. The Spitzer SAGE-SMC project was supported by NASA grant NAG512595. This work is based (in part) on archival data obtained with the Spitzer Space Telescope, which is operated by the Jet Propulsion Laboratory, California Institute of Technology under a contract with NASA. Support for this work was provided by an award issued by JPL/Caltech. This publication makes use of data products from the 2MASS, which is a joint project of the University of Massachusetts and the Infrared Processing and Analysis Center/California Institute of Technology, funded by the National Aeronautics and Space Administration and the National Science Foundation.

\section{Facility: Spitzer (IRAC, MIPS)}

\section{REFERENCES}

Antoniou, V., Hatzidimitriou, D., Zezas, A., \& Reig, P. 2009, ApJ, 707, 1080 Arp, B. H. 1959, AJ, 64, 254

Azzopardi, M., \& Vigneau, J. 1979, A\&AS, 35, 353

Azzopardi, M., \& Vigneau, J. 1982, A\&AS, 50, 291

Azzopardi, M., Vigneau, J., \& Macquet, M. 1975, A\&AS, 22, 285

Barbá, R. H., et al. 1995, ApJ, 446, L23

Bartzakos, P., Moffat, A. F. J., \& Niemela, V. S. 2001, MNRAS, 324, 18

Bolatto, A. D., et al. 2007, ApJ, 655, 212

Bonanos, A. Z., et al. 2009, AJ, 138, 1003

de Jager, C., Nieuwenhuijzen, H., \& van der Hucht, K. A. 1988, A\&AS, 72, 259

Dougherty, S. M., et al. 1994, A\&A, 290, 609

Dray, L. M. 2006, MNRAS, 370, 2079

Ekström, S., Meynet, G., Maeder, A., \& Barblan, F. 2008, A\&A, 478, 467

Evans, C. J., \& Howarth, I. D. 2008, MNRAS, 386, 826

Evans, C. J., et al. 2004a, MNRAS, 353, 601

Evans, C. J., et al. 2004b, ApJ, 607, 451

Evans, C. J., et al. 2006, A\&A, 456, 623

Feast, M. W., Thackeray, A. D., \& Wesselink, A. J. 1960, MNRAS, 121, 337

Foellmi, C., et al. 2008, RevMexAA, 44, 3

Foellmi, C., Moffat, A. F. J., \& Guerrero, M. A. 2003, MNRAS, 338, 360

Garmany, C. D., Conti, P. S., \& Massey, P. 1987, AJ, 93, 1070

Garmany, C. D., \& Humphreys, R. M. 1985, AJ, 90, 2009

Grebel, E. K., Richtler, T., \& de Boer, K. S. 1992, A\&A, 254, L5

Groenewegen, M. A. T. 2006, A\&A, 448, 181

Groenewegen, M. A. T., \& Blommaert, J. A. D. L. 1998, A\&A, 332, 25

Groenewegen, M. A. T., et al. 2009, A\&A, 506, 1277

Habing, H. J., Tignon, J., \& Tielens, A. G. G. M. 1994, A\&A, 286, 523

Harries, T. J., Hilditch, R. W., \& Howarth, I. D. 2003, MNRAS, 339, 157

Hilditch, R. W., Howarth, I. D., \& Harries, T. J. 2005, MNRAS, 357, 304

Humphreys, R. M. 1983, ApJ, 265, 176
Hunter, I., et al. 2007, A\&A, 466, 277

Ita, Y., et al. 2010a, A\&A, 514, 2

Ita, Y., et al. 2010b, PASJ, 62, 573

Kato, D., et al. 2007, PASJ, 59, 615

Kippenhahn, R., \& Weigert, A. 1990, Stellar Structure and Evolution (Berlin: Springer)

Lanz, T., \& Hubeny, I. 2003, ApJS, 146, 417

Lanz, T., \& Hubeny, I. 2007, ApJS, 169, 83

Larsen, S. S., Clausen, J. V., \& Storm, J. 2000, A\&A, 364, 455

Leitherer, C., Robert, C., \& Drissen, L. 1992, ApJ, 401, 596

Lennon, D. J. 1997, A\&A, 317, 871

Lennon, D. J., et al. 1993, Space Sci. Rev., 66, 169

Levesque, E. M., et al. 2006, ApJ, 645, 1102

Levesque, E. M., et al. 2007, ApJ, 667, 202

Liu, Q. Z., van Paradijs, J., \& van den Heuvel, E. P. J. 2005, A\&A, 442, 1135

Maeder, A., Grebel, E. K., \& Mermilliod, J. 1999, A\&A, 346, 459

Marshall, J. R., van Loon, J. T., Matsuura, M., Wood, P. R., Zijlstra, A. A., \& Whitelock, P. A. 2004, MNRAS, 355, 1348

Martayan, C., Baade, D., \& Fabregat, J. 2010, A\&A, 509, A11

Martayan, C., et al. 2007a, A\&A, 472, 577

Martayan, C., et al. 2007b, A\&A, 462, 683

Massey, P. 2002, ApJS, 141, 81

Massey, P. 2010, in ASP Conf. Ser. 425, Hot and Cool: Bridging Gaps in Massive Star Evolution, ed. C. Leitherer, et al., in press (arXiv:0903.0155)

Massey, P., \& Olsen, K. A. G. 2003, AJ, 126, 2867

Massey, P., Olsen, K. A. G., \& Parker, J. W. 2003, PASP, 115, 1265

Massey, P., Parker, J. W., \& Garmany, C. D. 1989, AJ, 98, 1305

Massey, P., Waterhouse, E., \& DeGioia-Eastwood, K. 2000, AJ, 119, 2214

Massey, P., et al. 1995, ApJ, 438, 188

Massey, P., et al. 2005, ApJ, 627, 477

Massey, P., et al. 2009, ApJ, 692, 618

McDonald, I., et al. 2009, MNRAS, 394, 831

Meixner, M., et al. 2006, AJ, 132, 2268

Mennickent, R. E., et al. 2006, A\&A, 457, 949

Mokiem, M. R., et al. 2007, A\&A, 473, 603

Negueruela, I., Steele, I. A., \& Bernabeu, G. 2004, Astron. Nachr., 325, 749

Oestreicher, M. O., Schmidt-Kaler, T., \& Wargau, W. 1997, MNRAS, 289, 729

Osmer, P. S. 1973, ApJ, 181, 327

Panagia, N., \& Felli, M. 1975, A\&A, 39, 1

Panagia, N., \& Thum, C. 1981, A\&A, 98, 295

Pojmański, G. 2002, Acta Astron., 52, 397

Porter, J. M., \& Rivinius, T. 2003, PASP, 115, 1153

Prieto, J. L., et al. 2008, ApJ, 673, L59

Raguzova, N. V., \& Popov, S. B. 2005, Astron. Astrophys. Trans., 24, 151

Reach, W. T., et al. 2005, PASP, 117, 978

Sanduleak, N. 1968, AJ, 73, 246

Sanduleak, N. 1969, AJ, 74, 877

Skrutskie, M. F., et al. 2006, AJ, 131, 1163

Sloan, G. C., et al. 2008, ApJ, 686, 1056

Smith Neubig, M. M., \& Bruhweiler, F. C. 1997, AJ, 114, 1951

Srinivasan, S., et al. 2009, AJ, 137, 4810

Szczygiel, D. M., et al. 2010, AJ, 140, 14

Szymański, M. K. 2005, Acta Astron., 55, 43

Udalski, A., Kubiak, M., \& Szymański, M. 1997, Acta Astron., 47, 319

Udalski, A., et al. 2008, Acta Astron., 58, 329

van Loon, J. T. 2000, A\&A, 354, 125

van Loon, J. T. , et al. 2006, in ASP Conf. Ser. 353, Stellar Evolution at Low Metallicity: Mass Loss, Explosions, Cosmology, ed. H. J. G. L. M. Lamers (San Francisco, CA: ASP), 211

van Loon, J. T. 2007, in ASP Conf. Ser. 378, Why Galaxies Care About AGB Stars: Their Importance as Actors and Probes, ed. F. Kerschbaum,

C. Charbonnel, \& R. F. Wing (San Francisco, CA: ASP), 227

van Loon, J. T., et al. 1999, A\&A, 351, 559

van Loon, J. T., et al. 2005, A\&A, 438, 273

van Loon, J. T., et al. 2008, A\&A, 487, 1055

van Loon, J. T., et al. 2010a, AJ, 139, 68

van Loon, J. T., et al. 2010b, AJ, 139, 1553

Verhoelst, T., et al. 2009, A\&A, 498, 127

Vink, J. S., de Koter, A., \& Lamers, H. J. G. L. M. 2001, A\&A, 369, 574

Wisniewski, J. P., \& Bjorkman, K. S. 2006, ApJ, 652, 458

Wisniewski, J. P., et al. 2007, ApJ, 670, 1331

Wood, P. R., et al. 1992, ApJ, 397, 552

Wright, A. E., \& Barlow, M. J. 1975, MNRAS, 170, 41

Zaritsky, D., et al. 2002, AJ, 123, 855

Zickgraf, F.-J. 2006, in ASP Conf. Ser. 355, Stars with the B[e] Phenomenon, ed. M. Kraus \& A. S. Miroshnichenko (San Francisco, CA: ASP), 135 